
Generalized Sub-Gaussian log-conductivity distributions

$5 \quad{ }^{1}$ Earth and Environmental Sciences Area, Geochemistry Department, Lawrence Berkeley

$6 \quad$ National Laboratory

$7 \quad{ }^{2}$ Department of Civil and Environmental Engineering (DECA), Universitat Politècnica 8 de Catalunya

Guillem Sole-Mari ${ }^{1,2,3}$, Monica Riva ${ }^{4}$, Daniel Fernàndez-Garcia ${ }^{2,3}$, Xavier SanchezVila $^{2,3}$, Alberto Guadagnini ${ }^{4}$

\section{${ }^{3}$ Associated Unit: Hydrogeology Group (UPC-CSIC)}

${ }^{4}$ Department of Civil and Environmental Engineering (DICA), Politecnico di Milano

\section{Highlights:}

- Log-conductivity $(Y)$ is modeled as a Generalized Sub-Gaussian (GSG) field

- Average longitudinal spreading is smaller for GSG than for Gaussian $Y$ fields

- Plume stretching in GSG fields is enhanced with respect to Gaussian fields

- Local dispersion masks identification of impacts of non-Gaussianity on transport

- The GSG nature of $Y$ can be difficult to identify relying only on transport metrics 
There are increasing evidences that probability distributions and associated statistical moments of a variety of hydrogeological and soil science variables and their spatial increments display distinctive scale-dependent features that are not captured by a typical Gaussian model. A Generalized Sub-Gaussian (GSG) model is able to capture key aspects of this pattern. We present the results of a suite of computational analyses set in a Monte Carlo framework and aimed at assessing the impact of a GSG structure of log hydraulic conductivity $(Y)$ on transport of a conservative solute through a threedimensional bounded porous medium under steady-state saturated Darcy flow. Our results indicate that the longitudinal spreading of a plume is on average significantly smaller for Sub-Gaussian than for Gaussian $Y$ fields. Otherwise, the velocity field arising from a Sub-Gaussian $Y$ field induces enhanced plume stretching with respect to what can be observed in a Gaussian $Y$ setting, this aspect potentially influencing the strength of solute mixing within these two types of conductivity domains. We also find that, in some cases, it may be difficult to identify the nature of the underlying conductivity field relying solely on observations of solute concentrations migrating within the system. In this regard, we show that the action of local dispersion tends to mask the influence of SubGaussianity on major transport metrics.

Keywords: Porous media, flow and transport, heterogeneity, stochastic modeling, SubGaussian models 
Flow and transport in porous and fractured media are strongly affected by spatial variability of hydraulic properties of the system. While it is virtually impossible to characterize all details of such heterogeneity, a large body of literature has framed the assessment of flow and transport processes taking place in heterogeneous porous media on a (geo)statistical description of continuum-scale system attributes, major emphasis being given on hydraulic conductivity (e.g., Dagan, 1984; Gelhar, 1993; Kitanidis, 1997; Rubin, 2003). Stochastic analyses of the effects of heterogeneity on flow and transport in porous media have been the subject of several investigations for over four decades. Among the variety of approaches developed in this context, studies embedded in a numerical Monte Carlo (MC) framework have enabled to explore subtle relationships between the heterogeneous features of the subsurface and salient elements characterizing flow and transport (e.g., Bellin et al., 1992; Naff et al., 1998a,b; Riva et al., 2008; de Dreuzy et al., 2012; Pedretti et al., 2017; Siena and Riva, 2018 and references therein). A typical Monte Carlo study comprises the analysis of a collection of outputs of interest that non-Gaussian features characterizing the distributions of a variety of hydrogeological and 
observation that the distribution of spatial increments of such variables taken between two points separated by a given spatial distance (lag) tend to be symmetric and to develop heavier tails and sharper peaks as lag decreases. Such a behavior has been displayed (among others) by log-hydraulic conductivity and permeability (Painter, 1996, 2001; Liu and Moltz, 1997; Meerschaert et al., 2004; Siena et al., 2012, 2019; Riva et al., 2013a, 2013b; Guadagnini et al., 2018), electrical resistivity (Painter, 2001), vadose zone hydraulic properties (Guadagnini et al., 2012, 2013, 2014), neutron porosity (Riva et al., 2015a), sediment transport (e.g., Ganti et al., 2009), fully developed turbulence (Boffetta et al., 2008), and micro-scale geochemical data (Siena et al., 2020).

The Generalized Sub-Gaussian (GSG) model introduced in the literature in the last few years (Riva et al., 2015a, 2015b; Panzeri et al., 2016) has been shown to be capable of capturing all of these aspects. According to the GSG model, the departure of the distribution of a variable and its two-point increments from the Gaussian one is given by the action of a (spatially uncorrelated) subordinator on an otherwise spatially correlated Gaussian random field. This modeling strategy allows representing jointly within a unique framework the above-documented behavior (as described by probability distributions and/or moments) of a quantity and its incremental values and has been successfully applied to the interpretation of main features displayed by various subsurface attributes (Riva et al 2015a; Guadagnini et al., 2018; Siena et al., 2020, and references therein).

These concepts have already been employed in preliminary analytical and numerical studies of flow and transport in porous media whose log-conductivity is characterized through a GSG model. Riva et al. (2017) present lead-order analytical flow and transport solutions in unbounded GSG log-conductivity fields under mean-uniform flow. Libera et al. (2017) rely on a numerical Monte Carlo framework to analyze the joint 
effects of a GSG heterogeneous log-conductivity field and a temporally variable pumping rate on solute breakthrough curves (BTCs) detected at a pumping well operating in a twodimensional domain. Besides spatial dimensionality, three major limitations can be identified for the analytical study by Riva et al. (2017): (i) macrodispersion is the only transport metric analyzed, (ii) the joint impact of local dispersivity combined with the heterogeneous advection driven by $Y$ is not evaluated, and (iii) output uncertainty due to finite size of the medium is not considered.

Starting from the above studies, here we focus on the influence of randomly heterogeneous GSG log-conductivity fields on transport processes taking place therein and address the following two questions: (1) At what extent does Sub-Gaussianity (i.e., the degree of departure from a Gaussian behavior) of $Y$ impact main features and observables of solute transport driven by advection and local dispersion in threedimensional bounded heterogeneous porous media?; and (2) Would the Sub-Gaussian nature of $Y$ be clearly identifiable upon relying solely on the analysis of solute concentration fields in these settings or could it be disguised into estimates of apparent structural parameters of a Gaussian model? To address these issues, here we rest on a suite of computational analyses framed in a Monte Carlo context and explore the effects of a Sub-Gaussian distribution of $Y$ on observable transport metrics associated with a solute migrating within a bounded three-dimensional porous medium under the action of advective and dispersive mechanisms and subject to a uniform (in the mean) flow.

The work is structured as follows. Section 2 is devoted to a synthesis of the theoretical framework underlying the Sub-Gaussian model and of the computational analyses performed. Presentation and discussion of the results are included in Section 3. Finally, in Section 4 we enumerate our main conclusions. 
$\ln K(\mathbf{x})$, is described as a stationary random function of space with constant ensemble mean, $\langle Y\rangle$, and zero-mean random fluctuation, $Y^{\prime}(\mathbf{x})=Y(\mathbf{x})-\langle Y\rangle$. The latter forms a Generalized Sub-Gaussian process, GSG, defined as (Riva et al. 2015a, b)

$$
Y^{\prime}(\mathbf{x})=U(\mathbf{x}) G(\mathbf{x})
$$

Here, $\mathbf{x}=[x, y, z]^{T}, G(\mathbf{x})$ is a zero-mean Gaussian spatially correlated second-order stationary field, and $U(\mathbf{x})$ is a random non-negative spatially uncorrelated stationary field that is independent of $G(\mathbf{x})$. The statistical properties of $Y^{\prime}(\mathbf{x})$ vary with the subordinator $U(\mathbf{x})$ and with the Gaussian field $G(\mathbf{x})$. Details about the mathematical formulation and theoretical framework of the GSG model can be found in Riva et al. (2015a) and Siena et statistical moments of $Y^{\prime}$ and of the corresponding increments $(\Delta Y)$ evaluated at various separation lags, including formulations for covariance and variogram functions as well as integral scale of $Y^{\prime}$. It is worth noting that, regardless the distributional form of $U$, the variogram of $Y^{\prime}$ is characterized by an integral scale which is always smaller than the one associated with the Gaussian field $G$ and a nugget effect, rendered by the product of the variance of $G$ and the variance of $U$.

Here, we follow Riva et al. (2015a, 2017) and consider Monte Carlo realizations of $Y^{\prime}(\mathbf{x})$ associated with a $\log$-normal subordinator, i.e., $\ln (U) \sim \mathcal{N}\left[0,(2-\alpha)^{2}\right], \alpha<2$. As such, $Y^{\prime}(\mathbf{x})$ is characterized by two constant parameters $\left(\alpha, \sigma_{G}^{2}\right)$ and a correlation function $\rho_{G} ; \sigma_{G}^{2}$ and $\rho_{G}$ being variance and correlation function of $G(\mathbf{x})$, respectively. Note that 
$Y^{\prime}(\mathbf{x})$ becomes Gaussian and coincides with $G(\mathbf{x})$ when $\alpha \rightarrow 2$. For completeness, Appendix A includes a brief summary of the key analytical formulations and properties of the GSG model we consider in this study.

We generate three-dimensional realizations of $Y(\mathbf{x})$ of size $L_{x} \times L_{y} \times L_{z}$ by setting $\langle Y\rangle=0, \sigma_{Y}^{2}=1$ (where $\left.\sigma_{Y}^{2}=e^{2(2-\alpha)^{2}} \sigma_{G}^{2}\right)$ and adopting an isotropic exponential correlation function for $G(\mathbf{x})$, i.e. $\rho_{G}=e^{-s / I_{G}} ; s$ and $I_{G}$ being the separation distance (lag) between two locations and the integral scale of $G(\mathbf{x})$, respectively. We set $L_{x}=$ $L_{y}=5 I_{Y}$ and $L_{z}=40 I_{Y}$ in all realizations $(z$ being the dominant direction of flow, see Section 2.2), and $I_{Y}=e^{-(2-\alpha)^{2}} I_{G}$ denoting the integral scale of $Y$ (see also Appendix A). The spatial grid employed for the generation involves 125,000 cubic blocks of size $I_{Y} / 5$. Figure 1 depicts examples of $Y(\mathbf{x})$ realizations obtained for differing values of $\alpha$. To appreciate the nature of the random log-conductivity fields considered, we illustrate in Appendix A the main features of the probability density functions associated with $Y^{\prime}$ and its spatial increments $\Delta Y$ evaluated at various separation lags for the fields depicted in Figure 1. Figure A1 depicts the probability density function of $Y^{\prime}, f_{Y^{\prime}}$, for the three values of $\alpha$ considered in Figure 1 together with the Gaussian $(\alpha \rightarrow 2)$ distribution. Corresponding $p d f_{s}$ of spatial increments, $f_{\Delta Y}$, evaluated at short, intermediate and large (with respect to $I_{Y}$ ) separation lags are depicted in Figure A2. The pattern associated with the behavior of peaks and tails of these $p d f s$ can be described quantitatively by analyzing deviations from Gaussianity, clearly revealed by the excess standardized kurtosis, reported in Figure A3. It is clear that the peak of the $p d f$ of $Y^{\prime}$ or $\Delta Y$ grows sharper and the associated tails become heavier as $\alpha$ decreases, thus evidencing the deviations of the fields we consider from a Gaussian behavior. 


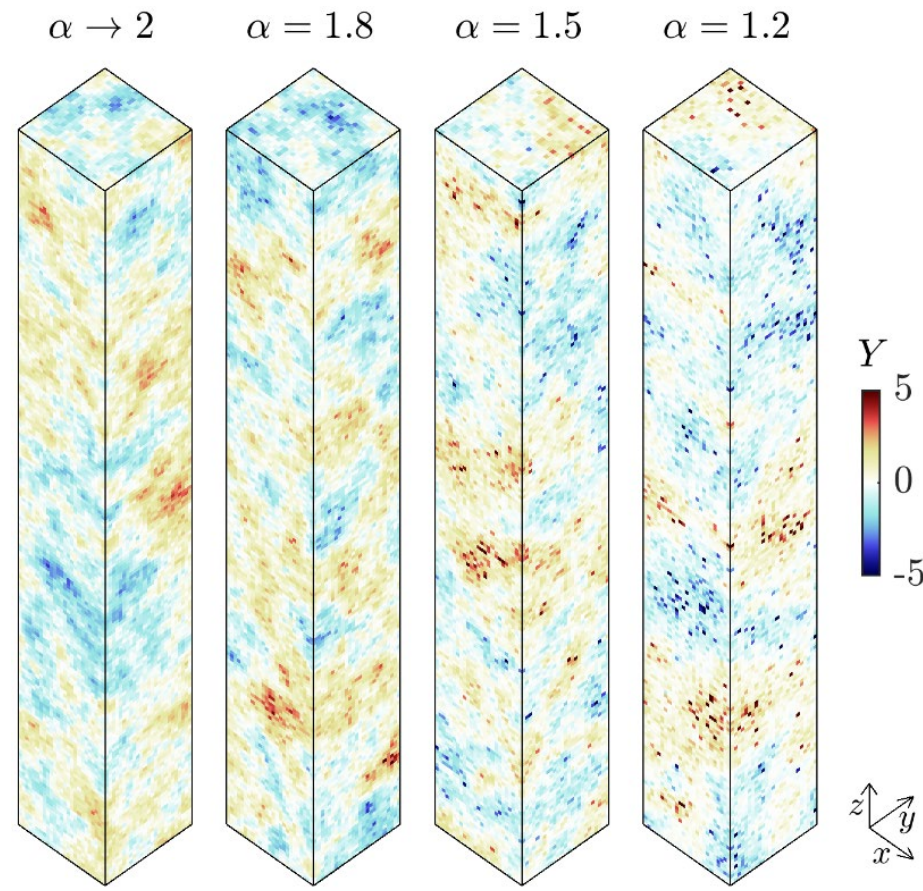

Figure 1: Example of $Y(\boldsymbol{x})$ individual realizations for various degrees of Sub-Gaussianity ( $\alpha \rightarrow 2$ being equivalent to a Gaussian distribution).

As a remark, we note that the variance of $Y$ in geologic porous media has sometimes been reported to attain markedly high values, e.g., $\sigma_{Y}^{2} \geq 4$ (Fogg \& Zhang, 2016). It is well recognized that this stems from a homogenization of conductivity values within a unique population, while they actually pertain to diverse geological facies. One may alternatively represent the system as a composite medium, whose internal architecture is characterized by the presence of distinct facies, within which hydraulic conductivity can be spatially heterogeneous. As such, conductivity variance within a given geomaterial can be mild (Winter \& Tartakovsky, 2000, 2002). Hence, the selected mild value of the log-conductivity variance in this work $\left(\sigma_{Y}^{2}=1\right)$ is deemed as representative of the natural variability contained within a geological unit, which can potentially be depicted through statistically stationary heterogeneity models (Winter et al., 2003) like the one we consider. The relative size of the cross-section (i.e., $L_{x}=L_{y}=5 I_{Y}$ ) is set to be large enough to capture the main features of the log-conductivity distributions, while being small enough 
to generate variability in the results between realizations with identical configuration, similar to what one would expect from a collection of finite-sized samples belonging to the same geological material or unit.

We further remark that here we focus on the analysis of the joint effects on transport of the strength of $(i)$ the departure of $Y$ from a Gaussian model (as rendered by the parameter $\alpha$ related to the subordinator $U$ ), and (ii) the relative importance of advective and dispersive processes in Sub-Gaussian $Y$ fields. Future studies can tackle also the potential influence of the degree of heterogeneity, as expressed in terms of $\sigma_{Y}^{2}$, or consider representing a log-conductivity field involving multiple subdomains, with diverse geomaterials through a multimodal distribution, such as a mixture of Sub-Gaussian $p d f s$.

\subsection{Flow and transport model}

Steady-state single phase flow is described through mass balance and Darcy's equations within the heterogeneous $K(\mathbf{x})$ fields generated as described in Section 2.1.

$$
\nabla \cdot \mathbf{q}(\mathbf{x})=0, \quad \mathbf{q}(\mathbf{x})=-K(\mathbf{x}) \nabla H(\mathbf{x}) .
$$

Here, $H(\mathbf{x})$ is hydraulic head and $\mathbf{q}(\mathbf{x})$ is Darcy flux. We set impermeable boundary conditions on the lateral sides of the domain $\left(x=0, L_{x} ; y=0, L_{y}\right)$. Flow is forced along direction $z$ by setting a constant mean flow, $\bar{q}$, at the bottom of the sample, and by setting a prescribed head at the top boundary. A homogeneous buffer zone of length $2 I_{Y}$ and with $Y=\langle Y\rangle$ is placed at both ends of the system (Figure 2) to stabilize the inflow condition. These boundary conditions correspond to what one would typically impose in an experimental set-up, head at the inlet being practically uniform in the transverse direction due to the presence of the homogeneous buffer region. Imposing a fixed mean longitudinal flow $\bar{q}$ for all realizations enables us to study transport phenomena which are driven by relative spatial fluctuations of $q(\mathbf{x})$ around the same mean value. 
At the initial time $(t=0)$ a flux-averaged pulse injection of solute takes place across the bottom of the heterogeneous region, i.e., along the horizontal plane $z=0$. Solute transport is described by the Advection Dispersion Equation (ADE)

$$
\frac{\partial c}{\partial t}(\mathbf{x}, t)=\boldsymbol{\nabla} \cdot(\mathbf{D}(\mathbf{x}) \boldsymbol{\nabla} c(\mathbf{x}, t))-\boldsymbol{\nabla} \cdot(\mathbf{v}(\mathbf{x}) c(\mathbf{x}, t))
$$

where $c(\mathbf{x}, t)$ is resident solute concentration (i.e., solute per unit volume of the porous medium), and $\mathbf{v}(\mathbf{x})=\mathbf{q}(\mathbf{x}) / \phi, \phi$ being porosity which we consider constant in this study. In the context of modeling transport in porous media, local dispersion is an upscaled apparent process that accounts for the combined effect of unresolved pore-scale velocity variations and molecular diffusion (e.g., Bear, 1972). At high pore-scale Péclet numbers, it is often considered to be proportional to the fluid velocity. To simplify the problem, we assumed an isotropic local dispersion tensor $\mathbf{D}(\mathbf{x})$,

$$
\mathbf{D}(\mathbf{x})=D(\mathbf{x}) \mathbf{1}_{3}, \quad D(\mathbf{x})=a|\mathbf{v}(\mathbf{x})|,
$$

where $\mathbf{1}_{3}$ is the $3 \times 3$ identity matrix, $a$ is a constant dispersivity, and $|\cdot|$ denotes the norm of a vector. The advection and dispersion timescales for a characteristic distance $I_{Y}$ can be compared through the Péclet number, $\mathrm{Pe}=I_{Y} / a$, higher values of Pe corresponding to a lower relative significance of the local dispersion process. In this study we explore settings associated with values of $\mathrm{Pe}$ ranging from $\mathrm{Pe}=10$ to $\mathrm{Pe} \rightarrow \infty$.

The steady-state flow problem (2) is solved by the widely tested finite difference code MODFLOW 2005 (Harabaugh, 2005) using the Preconditioned Conjugate Gradient method (PCG). The output cell-interface fluxes are then used to solve the ADE by means of the Random Walk Particle Tracking method suggested by LaBolle et al. (2000). The latter solves the so-called Generalized Stochastic Differential Equations (GSDE) and is based on the following particle movement 


$$
\mathbf{X}_{p}(t+\mathrm{d} t)=\mathbf{X}_{p}(t)+\mathbf{v}\left(\mathbf{X}_{p}(t)\right) \mathrm{d} t+\sqrt{2 D\left(\mathbf{X}_{p}(t)+\Lambda_{p}(t)\right) \mathrm{d} t} \boldsymbol{\xi}_{p}(t)
$$

$$
\boldsymbol{\Lambda}_{p}(t)=\sqrt{2 D\left(\mathbf{X}_{p}(t)\right) \mathrm{d} t} \boldsymbol{\xi}_{p}(t)
$$

where $\mathbf{X}_{p}(t)$ is the position of particle $p$ at time $t$, and $\xi_{p}(t)$ is a vector of 3 (the number of spatial dimensions) independent uncorrelated standard normal random numbers. Velocities at any point inside a numerical cell $\Omega$ are evaluated by linear interpolation

$$
\mathbf{v}(\mathbf{x} \in \Omega)=(1-\hat{\mathbf{x}}) \odot \mathbf{v}_{\Omega}^{-}+\hat{\mathbf{x}} \odot \mathbf{v}_{\Omega}^{+},
$$

where $\hat{\mathbf{x}}=\left(\mathbf{x}-\mathbf{x}_{\Omega}^{-}\right) \oslash\left(\mathbf{x}_{\Omega}^{+}-\mathbf{x}_{\Omega}^{-}\right)$; operators " $\odot ”$ and “ $\oslash$ " correspond to the elementwise Hadamard product and division, respectively; $\mathbf{x}_{\Omega}^{-}$and $\mathbf{x}_{\Omega}^{+}$are vectors containing the lower and upper cell interface location (for each direction); and $\mathbf{v}_{\Omega}^{-}$and $\mathbf{v}_{\Omega}^{+}$ are vectors whose entries are the corresponding cross-interface flow velocities.

The GSDE method (5) is particularly accurate and efficient when representing isotropic local dispersion characterized by strong local variations. Therefore, this method is suitable for reproducing transport features even within porous media characterized by local highly non-uniform flow conditions. We refer to Salamon et al. (2006) for a comprehensive review of random-walk methods for solving the advection-dispersion equation in spatially heterogeneous settings.

Solution of the flow problem (2) may require using a spatial grid finer than that introduced in Section 2.1 and according to which log-conductivity is generated. The grid block size, $\Delta$, as well as the number of particles, $n$, and the time step, $\Delta t$, considered for the implementation of the GSDE (5) have been selected as detailed in the following. The spatial grid resolution (i.e., $1 / \Delta$ ) and the number of particles $n$ are increased until convergence is attained, i.e., until no significant changes are observed in the numerical solution (as quantified through the metrics/indicators detailed in Section 3). The time step 
$\Delta t$ is adapted dynamically, to fulfill the condition which is most restrictive between $\Delta t \leq$ $\mathcal{C} \Delta /|\mathbf{v}|$ and $\Delta t \leq \mathcal{P}^{2} \Delta^{2} / 2 D, \mathcal{C}$ and $\mathcal{P}$ being numerical discretization parameters which are adjusted in the same fashion as $\Delta$ and $n$. The numerical model has been found to give an accurate solution of the flow and transport problem by setting $n=2^{18}(=262,144)$, $\mathcal{C}=\mathcal{P}=0.125$, and subdividing each conductivity block into 64 elements, i.e., $\Delta=$ $I_{Y} / 20$, giving rise to a computational grid formed by 8 million cells.

All particles are assigned an identical mass $M / n$ and are injected according to a flux-weighting scheme on the horizontal plane at $z=0$. Flow and transport are simulated across $N=5,000$ Monte Carlo realizations of $Y(\mathbf{x})($ see Section 2.1$)$ for $\alpha=1.2,1.5,1.8$, and $\alpha \rightarrow 2.0$ (i.e., Gaussian field), and for $\mathrm{Pe}=10,100,1000$, and $\mathrm{Pe} \rightarrow \infty$ (i.e., pure advection), for a total of 80,000 simulations. The latter have been performed using 180 cores from the Marconi A1 partition of the high-performance computer center CINECA (https://www.cineca.it/en). The total computational time required for our study is about 100,000 core hours.

In Section 3, we analyze the spatial and temporal moments, as well as additional key metrics (see also Appendix B for details), of

$$
C_{r}(z, t)=\frac{\phi}{M} \int_{0}^{L_{x}} \int_{0}^{L_{y}} C_{r}(\mathbf{x}, t) \mathrm{d} y \mathrm{~d} x
$$

$$
J_{r}(z, t)=\frac{\phi}{M} \int_{0}^{L_{x}} \int_{0}^{L_{y}} \mathbf{n}^{\mathrm{T}} \cdot\left[\mathbf{v}_{r}(\mathbf{x}) c_{r}(\mathbf{x}, t)-D_{r}(\mathbf{x}) \nabla c_{r}(\mathbf{x})\right] \mathrm{d} y \mathrm{~d} x,
$$

where $\mathbf{n}^{\mathrm{T}}=[0,0,1]$, and subscript $r$ refers to the realization number, i.e., $r=1, \ldots, N$. The quantity $C_{r}(z, t)$ is the longitudinal density of solute mass normalized by the total mass injected (hence it integrates to 1 along $z$ ). The quantity $J_{r}(z, t)$ is the net temporal flux of mass through a horizontal plane, also normalized by the total mass (hence it 
276 integrates to 1 with $t)$. Hereafter, we refer to $C_{r}(z, t)$ as the section-integral

277 concentrations, and to $J_{r}(z, t)$ as the breakthrough curve (BTC).

278 Results shown in Section 3 are illustrated in terms of dimensionless times, $t^{*}$, and 279 longitudinal positions, $z^{*}$, defined as

$$
t^{*}=\frac{\bar{v}}{I_{Y}} t, \quad z^{*}=\frac{Z}{I_{Y}}
$$

281 where $\bar{v}=\bar{q} / \phi$. Note that $t^{*}$ corresponds to the number of integral scales of $Y$ traversed 282 by the center of mass of the plume. The evolution of the solute plume is monitored by 283 recording particle positions at 20 increasing dimensionless times $t_{i}^{*}, i \in\{1,2, \ldots, 20\}$, 284 with $t_{1}^{*}=0.1, t_{20}^{*}=15$, and $t_{i}^{*}=t_{1}^{*} i^{\log _{20}\left(t_{20}^{*} / t_{1}^{*}\right)}$. It has been verified that all particles 285 are still located inside the heterogeneous domain in all MC realizations at $t_{20}^{*}$. We record 286 particle passage times through selected planes perpendicular to the mean flow direction 287 at distance $z_{j}^{*}\left(j \in\{1,2, \ldots, 20\}\right.$, with $z_{1}^{*}=0.2, z_{20}^{*}=30$, and $\left.z_{j}^{*}=z_{1}^{*} j^{\log _{20}\left(z_{20}^{*} / z_{1}^{*}\right)}\right)$ 288 from the origin. The design of the setup is depicted in Figure 2. 


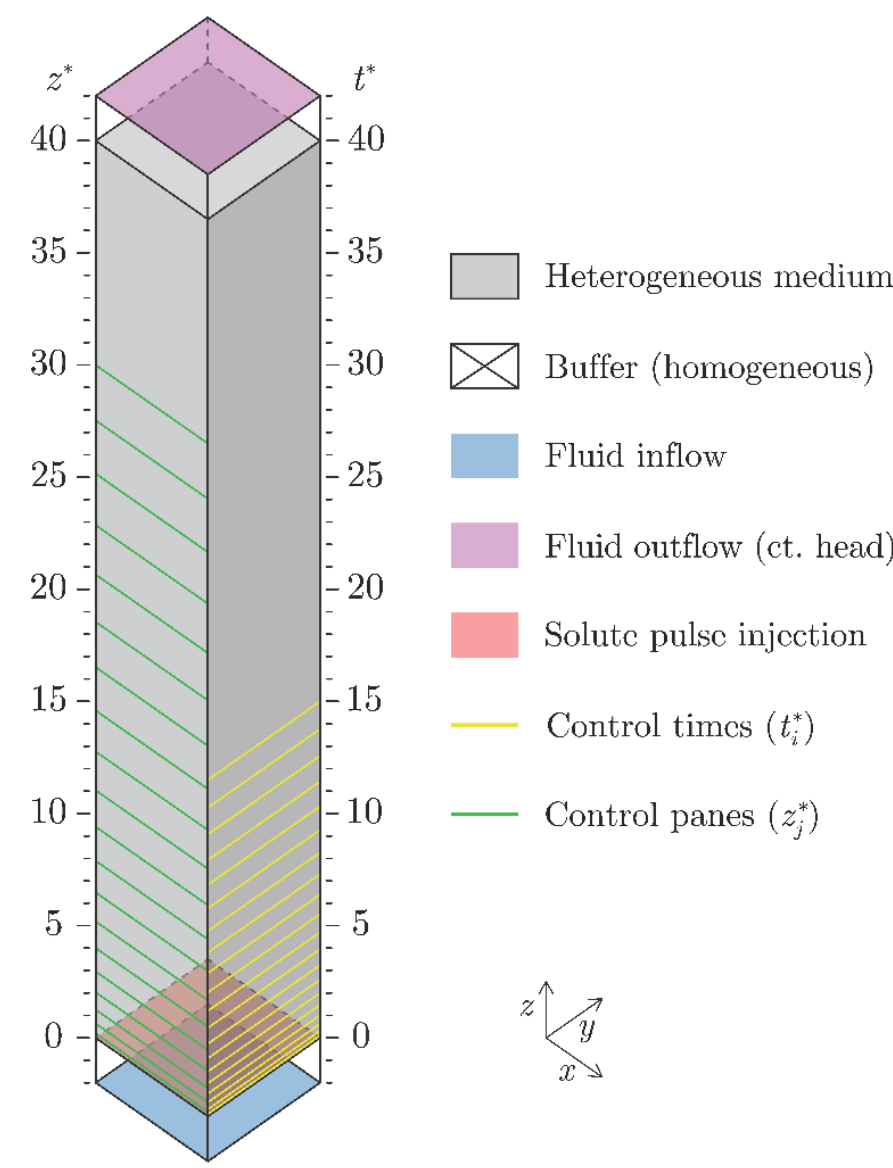

Figure 2: Design of the three-dimensional numerical simulations and scheme for recording the results. The control times are shown graphically in terms of the corresponding mean displacement of the fluid. Empty non-shaded regions at both ends correspond to the homogeneous buffers.

Our simulations yield a collection (obtained by sampling across $\mathrm{MC}$ realizations) of $(i)$ solute concentration profiles at a given observation time, $C_{r}\left(z, t_{i}\right)$, or (ii) solute breakthrough curves (BTCs) at a given transverse section, $J_{r}\left(z_{j}, t\right)$. We follow Liu and Müller (2004) and Lu and Stauffer (2012) and note that, in this context, a point-wise average is not a proper estimate of the mean concentration profile (or curve). For instance, such an average tends to overestimate the spread of the tails and may also underestimate the peak. This issue is addressed by employing a percentile average over the cumulative forms of all curves (see Lu and Stauffer (2012) for details). The shape of the ensuing average curves is generally more similar than the point-wise average to the one of curves 
corresponding to individual realizations. Here, we evaluate percentile averages $\bar{C}\left(z, t_{i}\right)$ arrival times, respectively) in increasing order, and then averaging across realizations, to finally reconstruct the density of data by Kernel Density Estimation (KDE). Details on this procedure are offered in Appendix B.1 (see also Appendix D for details on KDE). For brevity, hereafter we refer to $\bar{C}\left(z, t_{i}\right)$ and $\bar{J}\left(z_{j}, t\right)$ as the average longitudinal concentration profile and the average BTC, respectively. Note that these percentileaveraged ensemble quantities are denoted with an overline, as opposed to angular brackets (i.e., \langle\rangle$)$ indicating arithmetic ensemble average, which we employ for the scalar metrics analyzed (see eq. (11) in Section 3).

\section{Results and discussion}

We start by analyzing our results in terms of average spatial and temporal dynamics of the solute on the basis of the numerical Monte Carlo framework described in Section

2. We do so by considering the setting corresponding to pure advective mechanisms, i.e., $\mathrm{Pe} \rightarrow \infty$ (Section 3.1.1), to then include the assessment of the impact of considering a complete set of statistical distributions of the modeling goals of interest.

\subsection{Average space-time dynamics of the solute} $b$ of interest, as obtained across the collection of $N$ Monte Carlo realizations, i.e.,

$$
\langle b\rangle=\frac{1}{N} \sum_{r=1}^{N} b_{r},
$$

$b_{r}$ being the value of metric $b$ in the $r^{\text {th }}$ realization. 

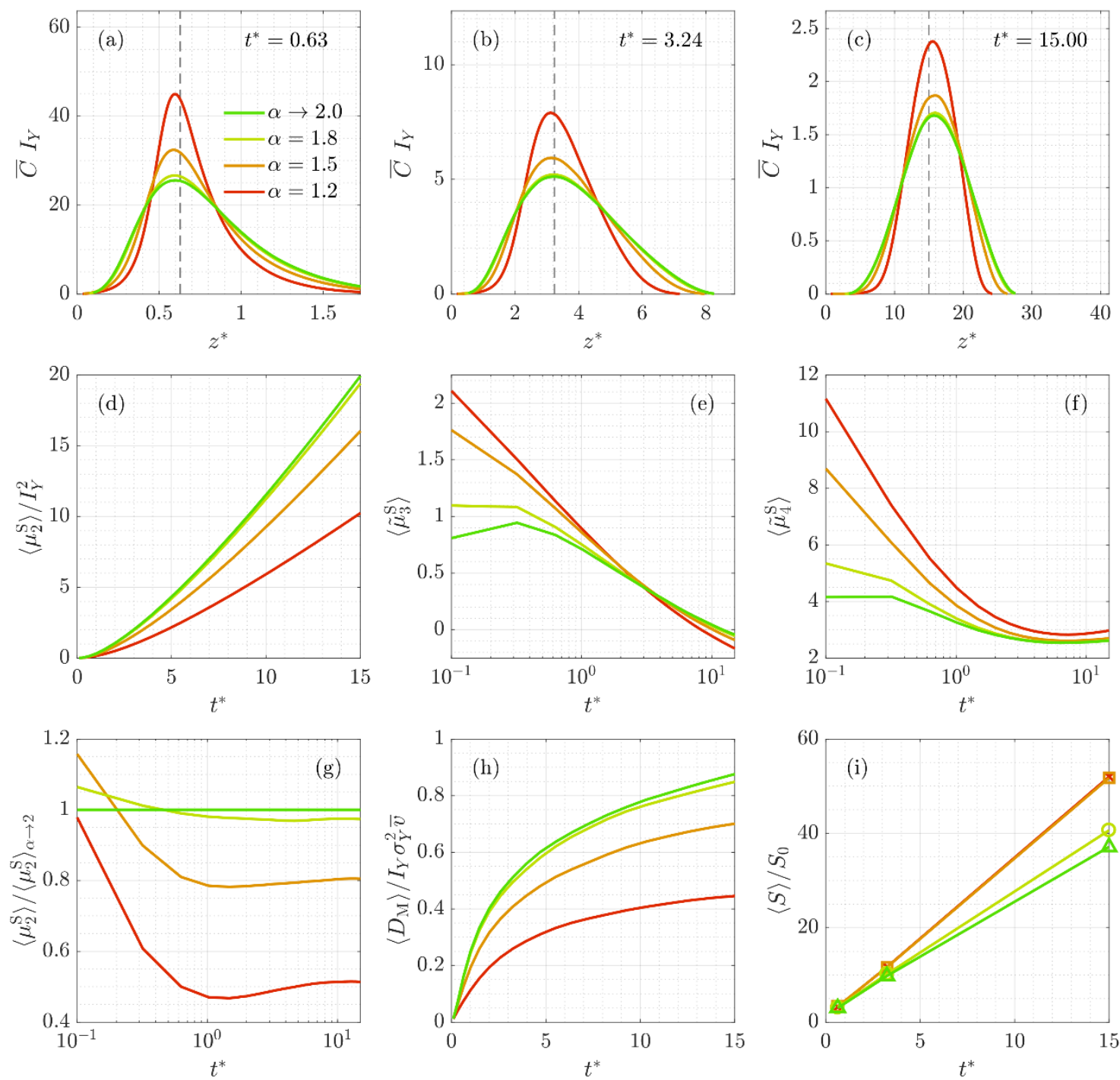

Figure 3: Average temporal evolution of the solute distribution in the longitudinal direction: (a-c) Longitudinal concentration profile at various times (dashed gray line corresponds to $z=\bar{v} t$ ), (d) spatial variance, (e) spatial skewness, $(f)$ spatial kurtosis, $(g)$ spatial variance normalized by the Gaussian case, (h) macrodispersion coefficient, and

Figures 3(a-c) depict the average longitudinal concentration profile, $\bar{C}$, evaluated as detailed in Section 2.2 and Appendix B.1, for various values of $\alpha$ at increasing values of $t^{*}$ (the vertical dashed gray lines correspond to the average fluid displacement, $z=\bar{v} t$ ). In the following, we discuss the effect of $\alpha$ on the temporal evolution of the plume by 
analyzing these concentration profiles in combination with various metrics of interest depicted within Figures 3(d-i).

The longitudinal extent of the region where one can find (on average) significant concentration values tends to decrease with departure of $Y$ from Gaussianity (i.e., decreasing $\alpha$ ). A quantification of this qualitative behavior can be offered through the average centered second moment of the longitudinal profile of section-integral concentrations, $\left\langle\mu_{2}^{\mathrm{S}}\right\rangle$, which is an effective measure of longitudinal dispersion (the latter being different from the local dispersion, as quantified by $\mathbf{D}$ in (3)), and whose temporal evolution is depicted in Figure 3(d). Details on the definition and computation of spatial and temporal moments in each MC realization are provided in Appendix B.2. Stronger degrees of Sub-Gaussianity (i.e., lower values of $\alpha$ ) are associated with decreased values of $\left\langle\mu_{2}^{\mathrm{S}}\right\rangle$. Figure $3(\mathrm{~g})$ also reveals that the ratio between any of the $\left\langle\mu_{2}^{\mathrm{S}}\right\rangle$ curves and the one corresponding to the Gaussian case $\left\langle\mu_{2}^{\mathrm{S}}\right\rangle_{\alpha \rightarrow 2}$ tends to become constant after a few characteristic times (i.e., for $\left.t^{*}>1\right)$. Quantities $1-\left\langle\mu_{2}^{\mathrm{S}}\right\rangle /\left\langle\mu_{2}^{\mathrm{S}}\right\rangle_{\alpha \rightarrow 2}$ and $(2-\alpha)^{2}$ display a nearly perfect linear correlation at late times (with a slope of $\sim 0.78$, details not shown). We note that dispersion in Gaussian $Y$ fields is, under some assumptions, proportional to $\sigma_{Y}^{2}$ (Dagan, 1984). As such, our results imply that, at long times, relying solely on an analysis of the macrodispersive behavior of a solute transported by pure advection within a saturated porous formation may not allow to distinguish whether the underlying log hydraulic conductivity distribution is $(i)$ Sub-Gaussian $(\alpha<2)$ with variance $\sigma_{Y}^{2}$, or $(i i)$ Gaussian with some variance $\sigma_{Y}^{* 2}$ being lower than $\sigma_{Y}^{2}$. Yet, as shown in the following, these two models lead to quite different estimates in a number of observables.

Figure 3(h) depicts the evolution of the average longitudinal (macro)dispersion coefficient, $\left\langle D_{\mathrm{M}}\right\rangle$, computed for each realization as 


$$
D_{\mathrm{M}, r}\left(t_{i}\right)=\left.\frac{1}{2} \frac{\partial \mu_{2, r}^{\mathrm{S}}}{\partial t}\right|_{t_{i}} \cong \frac{\mu_{2, r}^{\mathrm{S}}\left(t_{i}\right)-\mu_{2, r}^{\mathrm{S}}\left(t_{i-1}\right)}{2\left(t_{i}-t_{i-1}\right)}
$$

364 This quantity is normalized by $\sigma_{Y}^{2} I_{Y} \bar{v}$, which is the long-time asymptotic value of $D_{\mathrm{M}}$ in 365 an infinite medium with Gaussian log-conductivities characterized by low spatial heterogeneity. Note that an asymptotic dispersion regime is not fully reached by $t^{*}=15$. Riva et al. (2017) show that $D_{\mathrm{M}} / \sigma_{Y}^{2} I_{Y} \bar{v} \rightarrow 1$ (at a rate which depends on $\alpha$ ) in twodimensional Sub-Gaussian $Y$ fields and in the long-time limit, this result corresponding to analytical findings by Dagan $(1982,1984,1989)$ and Rubin $(1990 a, b)$ for Gaussian $Y$ fields under the same assumptions on flow. Assuming that a corresponding long-time limit holds also in three-dimensional unbounded systems, the results depicted in Figure 3(h) suggest that the impact of the presence of boundaries on the dynamics of $D_{\mathrm{M}}$ (i.e., on the departure of $D_{\mathrm{M}} / \sigma_{Y}^{2} I_{Y} \bar{v}$ from 1) tends to increase with decreasing $\alpha$.

Further inspection of Figures 3(a-c) reveals that low values of $\alpha$ yield a more pronounced peak for longitudinal concentration profiles. Additionally, the standardized distribution of vertical locations of the solute exhibits longer tails as $\alpha$ decreases (not shown). These features are markedly more evident at early times $\left(t^{*}=0.63\right)$. This result may be related to the observation that distributions of $Y$ increments (which are related to spatial variations of velocities, that ultimately control longitudinal solute spreading) in Sub-Gaussian fields display heavy tails and sharp peaks at short distances (Riva et al., 2015a). Differences between curves associated with differing values of $\alpha$ become less apparent at late times $\left(t^{*}=15\right)$. One can also detect an increasing degree of asymmetry of the plume as $\alpha$ decreases, both at early and late times.

All of these qualitative observations are quantified by the analysis of the temporal evolutions of the normalized spatial moments of the plume, namely the spatial skewness $386 \tilde{\mu}_{3}^{\mathrm{S}}$ (Figure 3(e)) and kurtosis $\tilde{\mu}_{4}^{\mathrm{S}}$ (Figure 3(f)) (see Appendix B.2 for details about their 
evaluation). We note that low $\alpha$ values tend to promote more skewed (i.e., asymmetric) plumes, being positively skewed at early times (a behavior mainly driven by high velocities) and (slightly) negatively skewed at late times (mainly driven by low velocities). Figure 3(f) suggests that lower values of $\alpha$ are associated (on average) with more kurtotic plumes. Differences between (average) spatial skewness and kurtosis of plumes evolving in Gaussian and Sub-Gaussian cases are substantial at early times and tend to become mild as time increases, consistent with the observation that the distribution of $Y$ spatial increments in a Sub-Gaussian field tends to approach the Gaussian one as lag increases.

Figure 3(i) depicts the temporal evolution of the average advective stretching, i.e., $\langle S\rangle / S_{0}$. This metric quantifies hydrodynamic deformation through the evaluation of the aggregated temporal growth of a fluid surface, $S$, represented by a collection of particles displaced by advection and originally arranged on the horizontal plane $z=0$ (i.e., corresponding to $S_{0}=L_{x} L_{y}$ ). All details are provided in Appendix B.4. On average, the velocity field associated with a Sub-Gaussian $Y$ field induces more stretched plumes than those evolving through a Gaussian setting. It is worth noting that this takes place despite the longitudinal spreading (represented by $\mu_{2}^{\mathrm{S}}$ ) being (sometimes significantly) smaller 404 for Sub-Gaussian than for Gaussian fields. One may compare these results with those reported by Le Borgne et al. $(2013,2015)$ in two-dimensional Gaussian $Y$ fields. Numerical simulations performed by these authors document that the mean elongation of a solute line displays a power-law temporal behavior with an exponent ranging between 0.65 and 1.25 for $\sigma_{Y}^{2}$ increasing from 0.25 to 4.0 . Interpreting the temporal evolution of $\langle S\rangle / S_{0}$ through a power-law behavior (as in the above-mentioned Gaussian $Y$ settings), 410 its (best-fit) exponent increases for decreasing values of $\alpha$ (while $\sigma_{Y}^{2}=1$ is kept 411 constant), from 0.85 in the Gaussian case to 0.95 for $\alpha \leq 1.5$. These results are probably 
412 related to the observation that low values of $\alpha$ promote marked changes in longitudinal 413 velocity values at very short transverse distances (details not shown here for brevity), thus 414 inducing enhanced degrees of short-range fluid stretching. This effect seems to be capped 415 at strong degrees of Sub-Gaussianity (i.e., $\alpha=1.2$ ), for which the average surface 416 increase is almost identical to the case of moderate-to-high Sub-Gaussian behavior (i.e., $417 \alpha=1.5)$.

418 The stark differences observed between the effects of a Sub-Gaussian $Y$ field on the 419 longitudinal dispersion (Figure 3(d)) and on advective stretching (Figure 3(i)) could 420 indicate that the increased longitudinal dispersion associated with Gaussian $Y$ fields with 421 respect to a Sub-Gaussian setting may not necessarily translate into enhanced mixing, the 422 latter being strongly linked to stretching (see, e.g., Dentz et al., 2011; Le Borgne et al., $4232013,2015)$. 

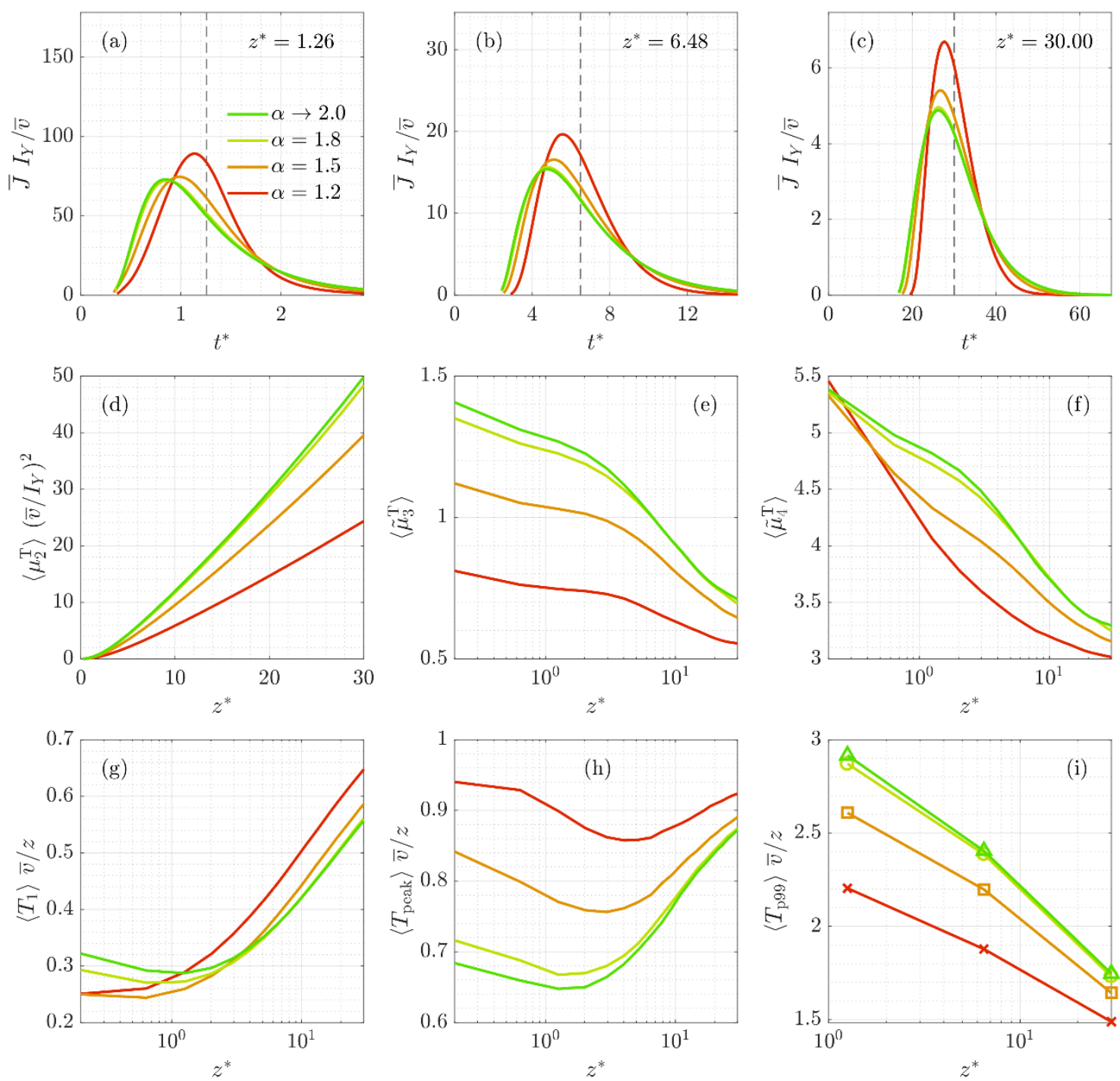

424

Figure 4: Average spatial evolution of the solute BTCs across planes orthogonal to the mean flow direction: (a-c) BTCS at various distances from the inlet (dashed gray line corresponds to $t=z / \bar{v}),(d)$ temporal variance, (e) temporal skewness, $(f)$ temporal kurtosis, (g) first arrival, (h) arrival of the peak, and (i) arrival of the 99\% of the total mass.

Figures $4(\mathrm{a}-\mathrm{c})$ depict the average temporal evolution of the solute mass flux, representing the solute breakthrough curve (BTC), for various values of $\alpha$ and at increasing distances from the inlet. The average BTCs are visibly narrower for lower values of $\alpha$. This is related to the observation that the spatial evolution of the centered second temporal moment, depicted in Figure 4(d), has been found to be qualitatively very 
similar to its temporal evolution counterpart (Figure 3(d)) in all cases analyzed, a feature which was also noted by Fernàndez-Garcia et al. (2005) for transport in threedimensional, Gaussian $Y$ fields. The average BTC is also visibly more symmetric for lower values of $\alpha$, the arrival of the peak being less advanced in time with respect to the mean arrival time $z / \bar{v}$ (marked on Figures $4(\mathrm{a}-\mathrm{c})$ by a gray vertical dashed line) than its counterpart based on Gaussian $Y$ fields. This latter feature is particularly evident at short distances from the inlet $\left(z^{*}=1.26\right)$.

For all values of $\alpha$, the BTCs become more symmetric as $z^{*}$ increases. This behavior can be quantified through the (average) skewness of the BTCs, i.e., $\left\langle\hat{\mu}_{3}^{\mathrm{T}}\right\rangle$, whose spatial evolution along the column (Figure 4(e)) denotes a decreasing trend of the temporal skewness $(i)$ with increasing distance from the inlet and (ii) with departure from Gaussian behavior of the underlying $Y$ field. A similar feature is observed in Figure 4(f) for the average temporal kurtosis, $\left\langle\hat{\mu}_{4}^{\mathrm{T}}\right\rangle$.

Figures 4(g), 4(h) and 4(i) complement the description by depicting the average longitudinal distribution of key arrival times, corresponding to the time of arrival of the first particle, $T_{1}$, the BTC peak, $T_{\text {peak }}$, and the $99^{\text {th }}$ percentile, $T_{\text {p99 }}$, respectively. These quantities are normalized by the mean arrival time (i.e., $z / \bar{v}$ ) for ease of interpretation. It is generally recognized that first arrival times are strongly linked to connectivity metrics, such as, e.g., the least resistance path, which is the path connecting two points or surfaces with the largest associated effective hydraulic conductivity (e.g., Gómez-Hernández and Wen, 1998; Rizzo and De Barros, 2017). In this context, Figure 4(g) suggests that SubGaussian $Y$ fields are more connected than their Gaussian counterparts at short distances $\left(z^{*} \leq 1\right)$, the trend being reversed at longer distances $\left(z^{*} \gg 1\right)$. This finding could be related to the aforementioned nugget effect displayed by the longitudinal velocity covariances associated with low $\alpha$ values, which would increase the likelihood of 
461 velocities changing abruptly at very short distances, and possibly decrease the likelihood

462 of occurrence of persistent high velocity paths.

463

As $\alpha$ decreases, the peak of the BTC (Figure 4(h)) is on average closer to the mean

464 arrival time, a finding in agreement with the aforementioned reduced temporal skewness.

465 This implies that in Sub-Gaussian fields the largest concentrations tend to be observed

466 (on average) appreciably later than in Gaussian fields, especially for short distances (i.e.,

$467 Z^{*} \approx 1$ ). The average longitudinal distribution of the $99^{\text {th }}$ percentile of arrival times, $T_{\mathrm{p} 99}$

468 (Figure 4(i)), shows that the BTC tends to be more compacted for lower values of $\alpha$. All

469 of these findings suggest that, as compared to a Gaussian distribution of log-

470 conductivities, in the presence of a Sub-Gaussian $Y$ field one can (on average) expect $(i)$

471 a generally delayed arrival of the mass of a flux-injected solute to crossing planes located

472 a few integral scales away from the injection plane (i.e., for $z^{*}>5$ ), as expressed in terms

473 of both the first arrival and the arrival of the peak; (ii) more pronounced peaks; and (iii)

474 an earlier breakthrough of the majority (99\%) of the solute mass. 

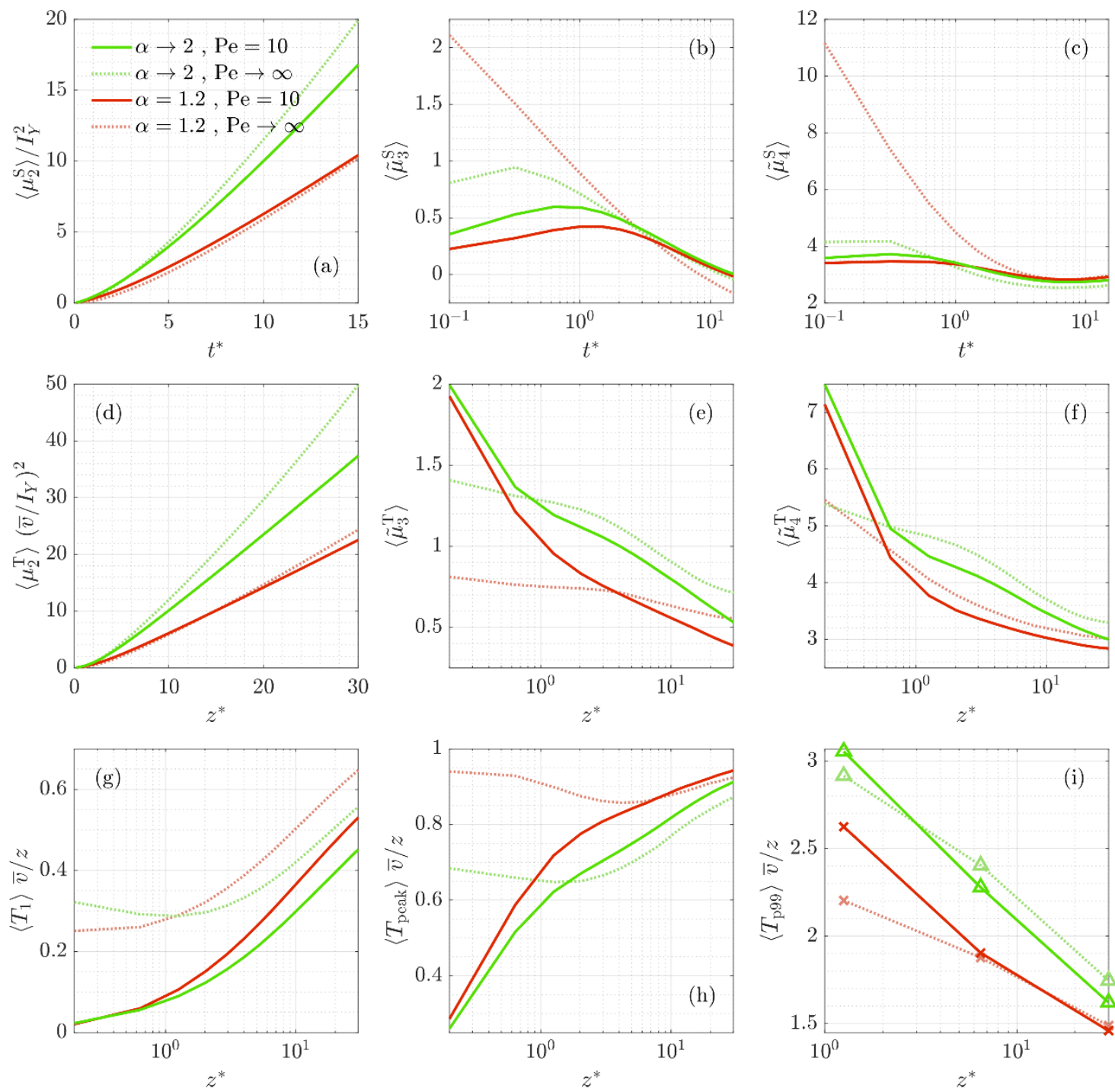

476

Figure 5: Effect of local dispersion $(P e=10)$ compared to pure advection $(P e \rightarrow \infty)$ on the results with Gaussian and Sub-Gaussian ( $\alpha=1.2)$ Y fields, for (a) spatial variance (macrodispersion), (b) spatial skewness, (c) spatial kurtosis, (d) temporal variance, (e) temporal skewness, (f) temporal kurtosis, ( $g$ ) first arrival, (h) arrival of the peak, and (i) arrival of the $99 \%$ of the solute mass.

Here, we concentrate on the scenario corresponding to the lowest value of $\alpha$ considered (i.e., $\alpha=1.2$; strongly non-Gaussian $Y$ ), compared to the Gaussian $Y$ scenario (i.e., $\alpha \rightarrow 2$ ), to clearly illustrate the effects of local dispersion (i.e., finite values of Pe) on transport. In Figure 5 we compare the spatial and temporal variance, skewness and kurtosis as well as the longitudinal distribution of key arrival times (defined in Section 
3.1.1) obtained for $\mathrm{Pe} \rightarrow \infty$ (dotted curves) with those corresponding to $\mathrm{Pe}=10$ (solid curves).

As one could expect, local dispersion typically reduces the difference between the results for Gaussian and Sub-Gaussian settings. In the Gaussian case (green curves), one can see that $\left\langle\mu_{2}^{\mathrm{S}}\right\rangle$ (Figure 5a) decreases due to the effect of local dispersion. This effect is increasingly evident for decreasing values of Pe (not shown). Such a behavior has been documented (analytically, numerically, and experimentally) in previous studies (e.g., Dentz et al., 2000, 2002, 2003; Dartois et al., 2018; Gist et al., 1990) and is related to the observation that local dispersion favors particles to sample a wider range of velocities, thus reducing the likelihood of sampling only extreme (low or high) velocity paths. Conversely, this effect is not apparent in the strongly Sub-Gaussian fields we examine. However, the aforementioned similarity between the temporal evolution of $\left\langle\mu_{2}^{\mathrm{S}}\right\rangle$ and the spatial evolution of $\left\langle\mu_{2}^{\mathrm{T}}\right\rangle$ and the results of Figure 5(d) suggest that the reduction in 501 longitudinal dispersion observed for $\mathrm{Pe}=10$ with respect to $\mathrm{Pe} \rightarrow \infty$ will eventually take 502 place in time also for $\alpha=1.2$.

While it was noted that the average longitudinal skewness of the plume, $\left\langle\tilde{\mu}_{3}^{\mathrm{S}}\right\rangle$, tends to deviate more strongly from zero both at early and late times as $\alpha$ decreases when $\mathrm{Pe} \rightarrow$ $\infty$, Figure 5(b) clearly documents that this trend is reversed at early times in the presence of local dispersion. A similar behavior is documented in Figure 5(c) for the average spatial kurtosis, $\left\langle\tilde{\mu}_{4}^{\mathrm{S}}\right\rangle$, the results corresponding to Gaussian and Sub-Gaussian settings tending to become very similar under the action of local dispersion. 
512 for $\alpha \rightarrow 2$ and $\alpha=1.2$. This feature is also observed for the first arrival time, $\left\langle T_{1}\right\rangle$.

513 Otherwise, the mass flux peak (Figure 5(h)) is delayed in time (on average) by the action

514 of a finite Pe, which also reduces the difference between the cases corresponding to $\alpha \rightarrow$

5152 and $\alpha=1.2$. The effect of dispersion on the (average) $99^{\text {th }}$ percentile arrival time,

$516\left\langle T_{\mathrm{p} 99}\right\rangle$, is analogous to that on $\left\langle\mu_{2}^{\mathrm{T}}\right\rangle$. As such, one can see that these two metrics and $\left\langle\mu_{2}^{\mathrm{S}}\right\rangle$

517 appear to contain very similar information about the effects of $\alpha$ and Pe on the processes

518 analyzed.

519

520

521

522

523

524

525

526

527

528

529

530

531

532

533

534

535

\subsection{Monte Carlo-based statistical distributions of metrics characterizing transport}

Here, we discuss the results obtained in terms of the full distributions of quantities of interest across the collection of Monte Carlo realizations analyzed. We focus on the system behavior at early and late stages of the plume deformation and confine the discussion to the scenarios corresponding to $\mathrm{Pe} \rightarrow \infty$ and $\mathrm{Pe}=10$. As a first result, we observe that the empirical MC-based distributions associated with most of the analyzed metrics are not well differentiated for the various values of $\alpha$ considered (i.e., there is some overlap between distributions). As such, we investigate the significance of relying on the metrics considered to discriminate the extent at which the log-conductivity field deviates from the Gaussian behavior (as reflected by the value of $\alpha$ ) when only results from a single realization of $Y$ are available. In other words, we analyze how significantly each metric of solute transport is impacted by the value of $\alpha$ characterizing a given $Y$ realization. We do so upon relying on the Bayes classifier (e.g., James et al., 2013) combined with Kernel Density Estimation.

Following the developments detailed in Appendix C, we quantify the degree of overlap between sample distributions of a given metric by means of the index $P_{k \ell}:=$ $P\left(\alpha=\alpha_{k} \mid \hat{\alpha}=\alpha_{\ell}\right)$, which represents the likelihood of the actual value of $\alpha$ being equal 
536 to $\alpha_{k}$, given that the classification method infers an estimate $\hat{\alpha}=\alpha_{\ell}$ (from comparing the

537 result of a realization to the corresponding Monte Carlo collection of results). We

538 consider the discrete set of $\alpha$ values corresponding to $\alpha_{k}, \alpha_{\ell} \in\{1.2,1.5,1.8, \rightarrow 2\}$. The

539 values of index $P_{k \ell}$ are then seen as the entries of a $(4 \times 4)$ matrix, which we refer to as

540 overlap matrix. Note that one can obtain an overlap matrix for each of the transport

541 metrics analyzed. Values on the diagonal (i.e., $P_{k \ell}$ with $k=\ell$ ) of this matrix indicate the

542 extent to which we can rely on the distribution of results for a given metric to identify the

543 actual value of $\alpha$ associated with the underlying $Y$ field. High values of the index

544 concentrated on the diagonal of the overlap matrix indicate mild overlap (i.e., strong

545 differentiation) of population densities, values evenly distributed across columns

546 suggesting strong overlap (i.e., poor differentiation). The strongest overlaps are observed

547 for high-order moments, whereas advective stretching is the best-differentiable metric.

548 For most metrics, overlap is strengthened at later times and for lower values of Pe. A

549 detailed analysis follows below. 

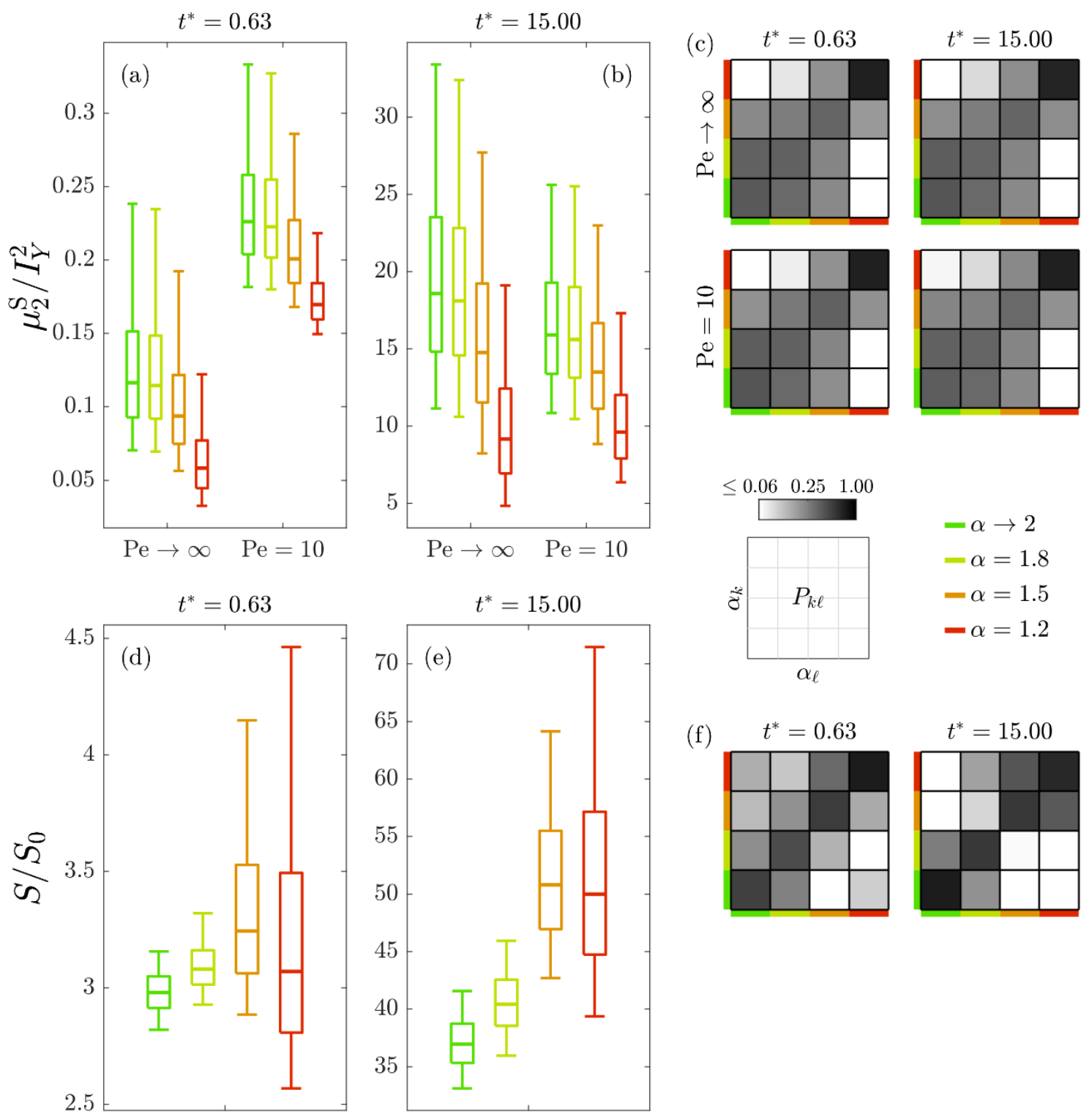

(f)

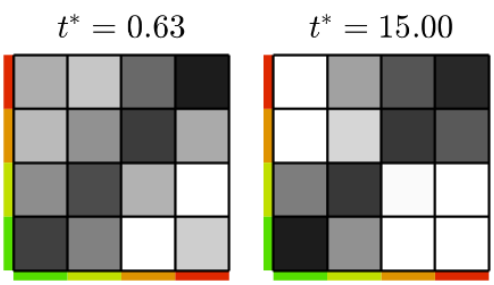

Figure 6: Box plot of (a)-(b) $\mu_{2}^{S}$ for $P e \rightarrow \infty$ and $P e=10$; and (d)-(e) $S / S_{0}$ for $P e \rightarrow \infty$ at included $(c, f)$.

Figure 6 depicts boxplots related to the populations of centered second moment of

556 the section-integral concentrations $\mu_{2}^{\mathrm{S}}$ (Figure $6(\mathrm{a}-\mathrm{b})$ ) and advective stretching $S / S_{0}$

557 (Figure 6(d-e)) for the various settings examined. These results are complemented by the graphical depiction of results stemming from the overlap analysis (see implementation details in Appendix C) for the two combinations of Pe (except for $S / S_{0}$, where $\mathrm{Pe} \rightarrow \infty$ 
560

561

562

563

564

565

566

567

568

569

570

571 obtained in the analysis of the populations and overlaps of $\mu_{2}^{\mathrm{T}}$ and $T_{\mathrm{p} 99}$ (not shown here

573

574 Gaussianity, this metric being the most affected by slight differences in $\alpha$. This is also

575 evidenced by the value of $P_{k \ell}$ in Figure 6(f). At late times $\left(t^{*}=15\right)$, there is only a slight

576

577 Gaussian / slightly Sub-Gaussian cases $(\alpha \geq 1.8)$. 

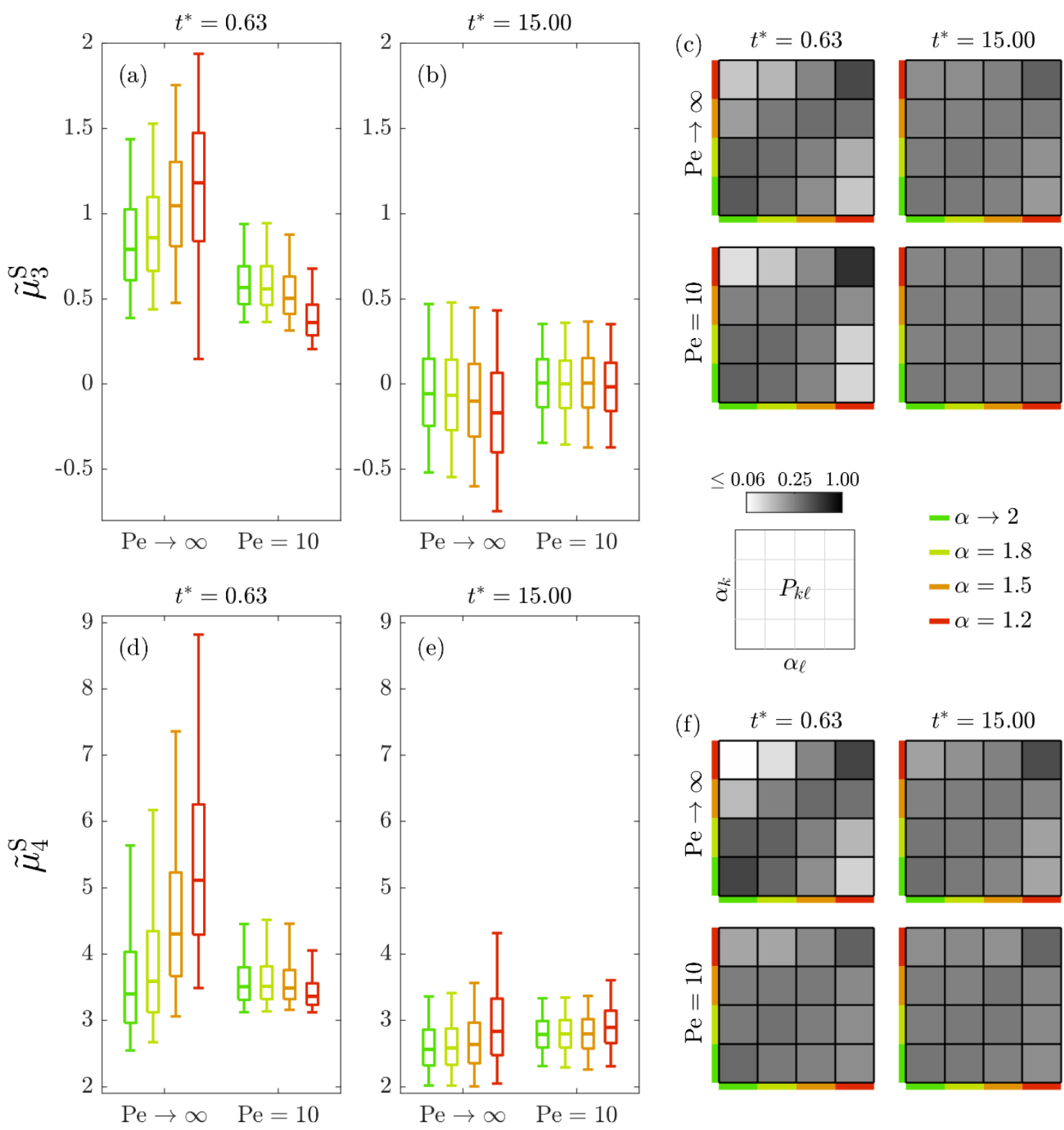

$$
\begin{aligned}
& -\alpha \rightarrow 2 \\
& -\alpha=1.8 \\
& -\alpha=1.5 \\
& -\alpha=1.2
\end{aligned}
$$
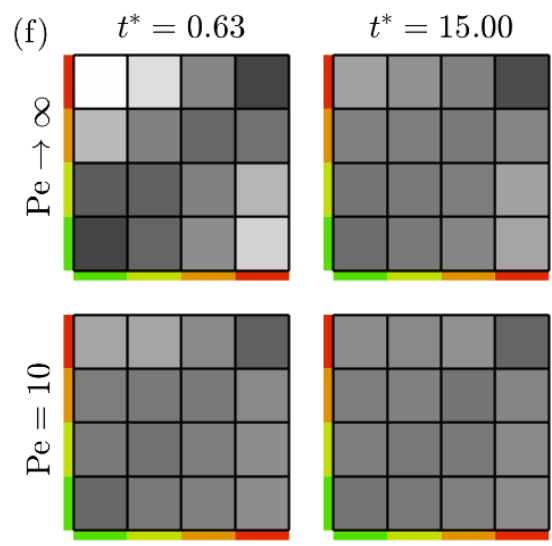

Figure 7: Box plot of (a)-(b) $\tilde{\mu}_{3}^{S}$ and (d)-(e) $\tilde{\mu}_{4}^{S}$ for $P e \rightarrow \infty$ and $P e=10$ at an early and at a late time. The corresponding overlap quantification, $P_{k \ell}$, is also included $(c, f)$.

Similar to what is observed for the centered second moment of the longitudinal concentration profile, a finite value of Pe narrows the range of values undertaken by the higher-order spatial moments across the collection of MC realizations (Figure 7). One can see that the distribution overlaps are significant (even for strongly Sub-Gaussian fields) for both skewness and kurtosis, especially at late times $\left(t^{*}=15\right)$ and for $\mathrm{Pe}=10$.

587 These results lead us to conclude that the average behavior of the higher-order moments 

probably be undetectable from the analysis of the evolution of solute concentrations across individual realizations of the $Y$ field. Similar conclusions are drawn from the analysis of the populations of higher-order temporal moments (not shown).
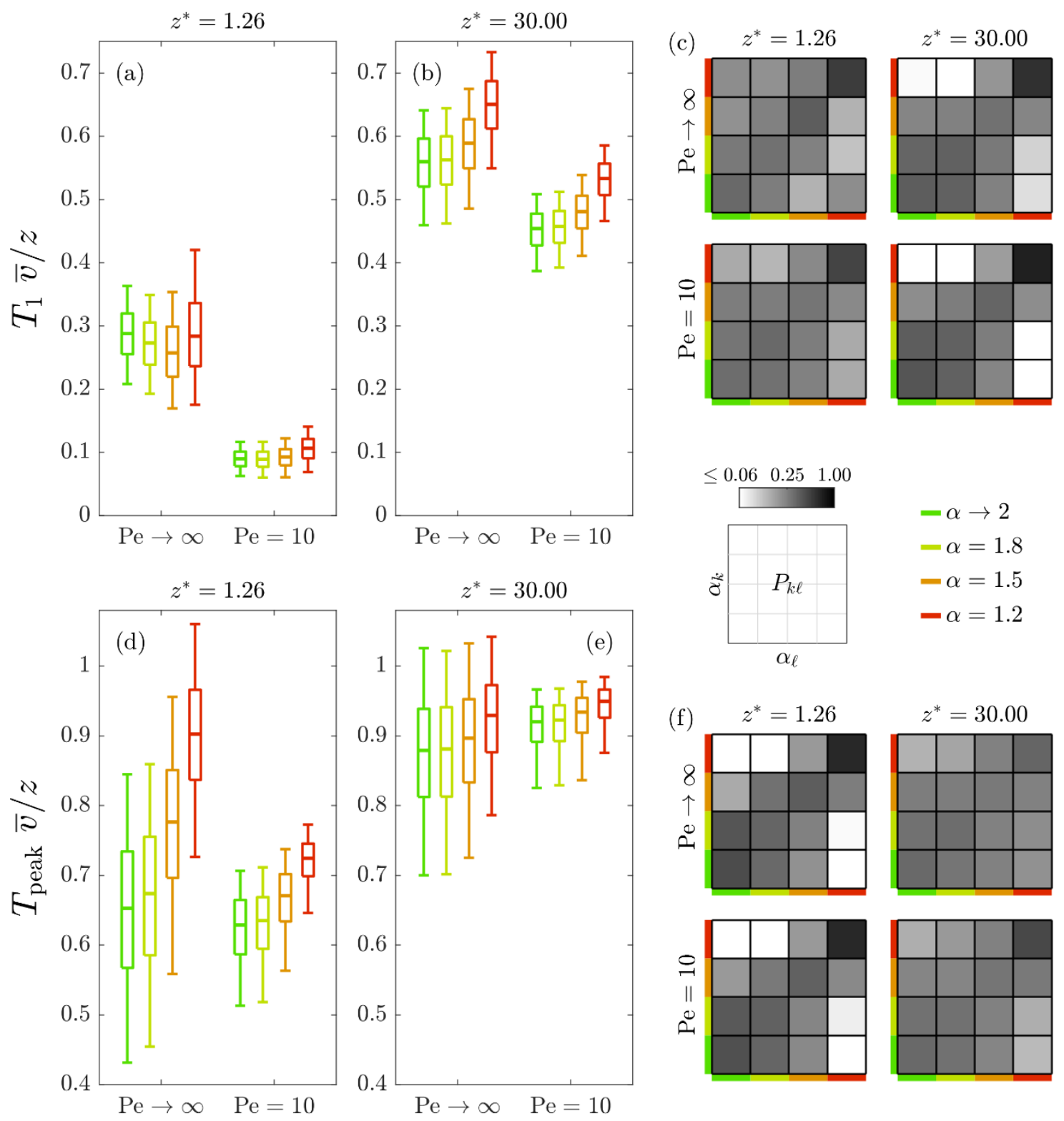

$$
\begin{aligned}
-\alpha & \rightarrow 2 \\
-\alpha & =1.8 \\
-\alpha & =1.5 \\
-\alpha & =1.2
\end{aligned}
$$
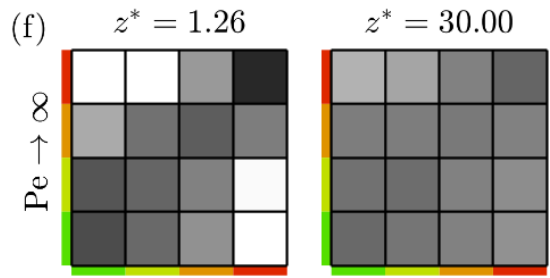

592

Figure 8: Box plot of (a)-(b) $T_{1}$ and (d)-(e) $T_{\text {peak }}$ for $P e \rightarrow \infty$ and $\mathrm{Pe}=10$ at a close and at a far distance from the inlet. The corresponding overlap quantification, $P_{k \ell}$, is also
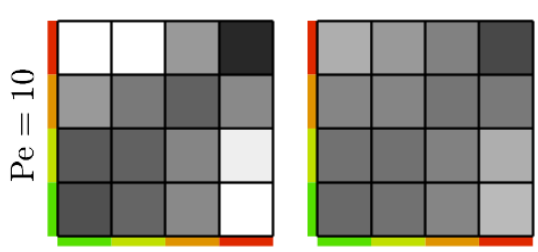
included $(c, f)$.

Figure 8 depicts the boxplots and overlap quantification corresponding to the first arrival time $\left(T_{1}\right)$ and the peak arrival time $\left(T_{\text {peak }}\right)$. At close distance from the inlet $\left(z^{*}=\right.$ 
598

599

600

601

602

603

604

605

606

607

608

609

610

611

612

613

614

615

616

617

618

619

1.26), the range of values of $T_{1}(i)$ narrows due to the effect of a finite Pe and (ii) is virtually insensitive to the value of $\alpha$. These results suggest that the behavior of $T_{1}$ is chiefly dominated by local dispersion, this impact somewhat persisting for long travel distances (i.e., $z^{*}=30$ ). These results support the conjecture that, even as the overlap with respect to the Gaussian case is not very high at long distances for moderate to strong Sub-Gaussian $Y$ fields, it would be hard to discriminate the effect of $\alpha$ from that of Pe if both parameters are not known a priori. It is also worth noting that, while the time at which the BTC peak occurs is (on average) closer to the mean travel time as $\alpha$ decreases (Section 3.1), this tendency can hardly be detected at long distances from single realizations. This is clearly seen in Figure 8(f), which shows that the overlap is nearly total at $z^{*}=30$.

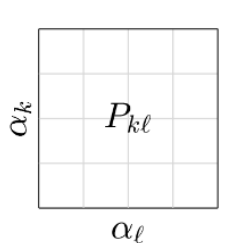

$-\alpha \rightarrow 2$

$-\alpha=1.8$

$-\alpha=1.5$

$-\alpha=1.2$

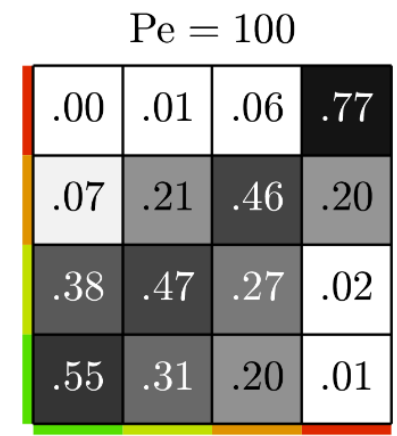

\begin{tabular}{|c|c|c|c|}
\multicolumn{5}{c|}{$\mathrm{Pe}=10$} \\
\hline .01 & .02 & .07 & .56 \\
\hline .12 & .21 & .34 & .26 \\
\hline .37 & .45 & .31 & .10 \\
\hline .50 & .32 & .29 & .08 \\
\hline
\end{tabular}

Figure 9: Overall overlap quantification, $P_{k \ell}$, between the distributions of the results corresponding to various values of $\alpha$ and for three selected Péclet numbers. The overlap is defined as $P_{k \ell}:=P\left(\alpha=\alpha_{k} \mid \hat{\alpha}=\alpha_{\ell}\right)$, where $\hat{\alpha}$ in this case is the result of a Quadratic Discriminant Analysis (see Appendix $C$ ) combining the information embedded in the evolution of all spatial and temporal moments as well as $T_{1}, T_{p e a k}$, and $T_{p 99}$.

Our analyses suggest that metrics that would typically be measurable in a real setting, such as the (spatial or temporal) moments describing the evolution of the plume, or the key arrival times, would not be able by themselves (i.e., without relying on additional observations about the $Y$ field) to reflect with certainty the Sub-Gaussian (or 
Gaussian) nature of the heterogeneous porous medium across which solute migrates. This is evidenced even assuming that $\sigma_{Y}^{2}, I_{Y}$, and Pe are known.

We further explore this aspect by analyzing the distributions of the results obtained for all of these metrics jointly. We do so by relying on the classification technique known as Quadratic Discriminant Analysis (QDA) to obtain an overall quantification of the degree of differentiation of the sample distributions of the multiple metrics considered.

Details about the theoretical elements underpinning the approach are illustrated in Appendix C. We ground QDA on the overall collections of results pertaining to spatial and temporal moments $\left(\mu_{2}^{\mathrm{S}}, \tilde{\mu}_{3}^{\mathrm{S}}, \tilde{\mu}_{4}^{\mathrm{S}}, \mu_{2}^{\mathrm{T}}, \tilde{\mu}_{3}^{\mathrm{T}}, \tilde{\mu}_{4}^{\mathrm{T}}\right)$, as well as the arrival times $T_{1}, T_{\text {peak }}$ and $T_{\mathrm{p} 99}$, and jointly considering results for $t^{*}=0.63,3.24,15$ and $z^{*}=1.26,6.48,30$, for a total of 27 sample populations. We exclude $S / S_{0}$ from the analysis, as we focus on solute transport metrics that could be detected directly in a real setting. This type of analysis yields a global appraisal of our ability to discriminate results associated with a unique realization through the assessment of an overlap matrix whose entries $P_{k \ell}$ represent the likelihood of the actual value of $\alpha$ being equal to $\alpha_{k}$ when the QDA classification yields a value equal to $\alpha_{\ell}$. To broaden the range of scenarios, we perform this analysis for $\mathrm{Pe}=10,100$, and $\mathrm{Pe} \rightarrow \infty$. The results are depicted in Figure 9. The degree of differentiation between the overall behavior of individual realizations is visibly higher than what one could infer by considering each of the metrics separately (Figures 6-8). Nevertheless, incorrect classifications are still abundant, especially for low values of Pe. The results of our joint analysis of spatial moments, temporal moments and key arrival times at multiple stages further support the observation that relying solely on observations of concentrations and/or travel times for the considered sample size does not allow to clearly and uniquely discriminate between values of $\alpha$. 

al. (2017), who found that manifestations of $Y$-field Sub-Gaussianity on moments/metrics associated with flow and transport tend to become virtually undetectable for $\alpha \geq 1.8$. This implies that $(i)$ the Gaussian model may suffice to reproduce some observable transport features even when the nature of the underlying $Y$ field is actually Sub-Gaussian, and (ii) it may be difficult in some cases to discern between non-Gaussian and Gaussian $Y$ fields based solely on transport metrics. These conclusions are also in line with results obtained by Jankovic et al. (2017), who analyzed breakthrough curves resulting from transport of solute plumes through porous media with spatial distributions of logconductivities relying on Gaussian and on various non-Gaussian statistical models. These authors concluded that the overall pattern of solute transport was relatively insensitive to the stochastic model employed, and that BTCs could be reasonably interpreted by relying solely on the knowledge of the mean, variance and integral scale of $Y$. As a consequence, we strengthen the concept that one is required to include a joint analysis of $Y$ data and their increments (as suggested by Riva et al. (2015a) or Guadagnini et al. (2018)) in efforts aimed at heterogeneity characterization to detect possible signatures supporting an appropriate stochastic model representing the nature of the underlying $Y$ field.

\section{Conclusions}

662

With reference to the assessment of the extent at which Sub-Gaussianity (i.e., the degree of departure from a Gaussian behavior) of $Y$ can impact main features and observables of solute transport driven by advection and local dispersion in threedimensional bounded heterogeneous porous media, we observe the following major elements. 
- As compared to a Gaussian distribution of log-conductivities, a Generalized SubGaussian (GSG) field of $Y$ yields (on average) ( $i$ ) a delayed first time of arrival of the solute mass to crossing planes located at a distance of a few integral scales from the solute injection plane; (ii) enhanced and delayed concentration peaks; and (iii) a reduced breakthrough time associated with the majority of the solute mass migrating in the system.

- On average, we document $(i)$ an increasing degree of asymmetry and (ii) an enhanced kurtosis (resulting in heavier tails) of the plume with log-conductivity departing from a Gaussian behavior. Differences between the effects imprinted by Gaussian and strongly Sub-Gaussian $(\alpha \leq 1.5) Y$ domains on spatial skewness and kurtosis of evolving plumes are marked at early times and tend to become mild as time increases.

- The velocity field associated with a GSG $Y$ field promotes plume stretching while reducing longitudinal plume spreading compared to its Gaussian counterpart. Therefore, the increased longitudinal dispersion associated with Gaussian $Y$ fields with respect to a GSG setting may not necessarily translate into enhanced solute mixing. This finding can have important implications on reactive transport; for example, in the context of mixing-limited reactive transport, it might imply that a higher mixing rate and reactivity can take place in Sub-Gaussian fields as compared to their Gaussian counterparts. Detailed analyses of this aspect will be subject of future research.

Concerning one's ability to clearly identify a possible underlying Sub-Gaussian nature of $Y$ upon relying solely on the analysis of solute concentration fields in the settings analyzed, our study leads to the following major conclusions. 
- Even as one has information about the values of variance and integral scale of $Y$, the effects of a Sub-Gaussian nature of $Y$ on hydrodynamic dispersion might hardly be detectable in a (finite) heterogeneous setting, especially if characterized by a low degree of Sub-Gaussianity (i.e., corresponding to $1.8 \leq \alpha<2.0$ for the type of subordinator considered). The action of local dispersion (i.e., finite values of Pe) can then contribute to further mask the influence of Sub-Gaussianity on major transport metrics, as also seen from a Quadratic Discriminant Analysis performed on spatial and temporal moments and arrival times at various evolution stages of the plume.

- While a GSG-based model can be consistent with scaling patterns of the probability distribution of log-conductivity and its spatial increments at various lags, relying solely on observations of solute concentrations migrating within the system can in some cases hamper our ability to discern the nature of the underlying conductivity field. To characterize the latter, one would need to consider also a joint analysis of $Y$ data and their increments.

We note that the present study focuses on mildly heterogeneous bounded domains (representing a spatially heterogeneous single geological unit) and is mostly based on spatially or temporally aggregated transport metrics. Additional studies are required to fully assess the extent to which the emergence of strictly local features associated with transport behavior (such as those related to strong channeling effects within highly heterogeneous, virtually unbounded media) might contribute to distinguish GSG logconductivity domains from their Gaussian counterparts.

\section{Acknowledgements}

The authors would like to thank the EU, MIUR, and MINECO for funding, in the frame of the collaborative international Consortium (WE-NEED) financed under the ERA-NET 
WaterWorks2014 Cofunded Call. This ERA-NET is an integral part of the 2015 Joint

Activities developed by the Water Challenges for a Changing World Joint Programme Initiative (Water JPI). Part of the work was developed while Prof. A. Guadagnini was at the University of Strasbourg with funding from Région Grand-Est and StrasbourgEurométropole through the Chaire Gutenberg. The UPC authors acknowledge funding from AGAUR Research Groups, 2017 SGR 1485.

Data available on Zenodo (https://doi.org/10.5281/zenodo.3356708).

\section{References}

Bear, J. (1972). Dynamics of fluids in porous media. Elsevier, New York.

Bellin, A., Salandin, P., \& Rinaldo, A. (1992). Simulation of dispersion in heterogeneous porous formations: Statistics, first-order theories, convergence of computations. Water Resources Research, 28(9), 2211-2227. https://doi.org/10.1029/92WR00578

Boffetta, G., Mazzino,A., \& Vulpiani, A. (2008). Twenty-five years of multifractals in fully developed turbulence: A tribute to Giovanni Paladin, J. Phys. A Math. Theor., 41,363001

Botev, Z. I., Grotowski, J. F., \& Kroese, D. P. (2010). Kernel density estimation via diffusion. Ann. Statist., 38(5), 2916-2957. https://doi.org/10.1214/10-AOS799

Dagan, G. (1982). Stochastic modeling of groundwater flow by unconditional and conditional probabilities: 2. The solute transport. Water Resources Research, 18(4), 835-848. https://doi.org/10.1029/WR018i004p00835

Dagan, G. (1984). Solute transport in heterogeneous porous formations. Journal of Fluid Mechanics, 145, 151-177. https://doi.org/10.1017/S0022112084002858

Dagan, G. (1989). Solute transport in porous formations. Springer, Berlin.

Dartois, A., Beaudoin, A., \& Huberson, S. (2018). Impact of local diffusion on macroscopic dispersion in three-dimensional porous media. Comptes Rendus Mécanique, 346(2), 89-97. https://doi.org/10.1016/j.crme.2017.12.012

de Dreuzy, J.-R., Méheust, Y., \& Pichot, G. (2012). Influence of fracture scale heterogeneity on the flow properties of three-dimensional discrete fracture networks (DFN). Journal of Geophysical Research: Solid Earth, 117(B11). https://doi.org/10.1029/2012JB009461 
Dentz, M., Kinzelbach, H., Attinger, S., \& Kinzelbach, W. (2003). Numerical studies of the transport behavior of a passive solute in a two-dimensional incompressible random flow field. Physical Review E - Statistical Physics, Plasmas, Fluids, and Related Interdisciplinary Topics. https://doi.org/10.1103/PhysRevE.67.046306

Dentz, M., Kinzelbach, H., Attinger, S., \& Kinzelbach, W. (2000). Temporal behavior of a solute cloud in a heterogeneous porous medium: 1. Point-like injection. Water Resources Research, 36(12), 3591-3604. https://doi.org/10.1029/2000WR900162

Dentz, M., Kinzelbach, H., Attinger, S., \& Kinzelbach, W. (2002). Temporal behavior of a solute cloud in a heterogeneous porous medium 3. Numerical simulations. Water Resources Research, 38(7), 13-23. https://doi.org/10.1029/2001WR000436

Dentz, M., Le Borgne, T., Englert, A., \& Bijeljic, B. (2011). Mixing, spreading and reaction in heterogeneous media: A brief review. Journal of Contaminant Hydrology. https://doi.org/10.1016/j.jconhyd.2010.05.002

Fernàndez-Garcia, D., \& Sanchez-Vila, X. (2011). Optimal reconstruction of concentrations, gradients and reaction rates from particle distributions. Journal of Contaminant Hydrology, 120-121, 99-114. https://doi.org/10.1016/j.jconhyd.2010.05.001

Fernàndez-Garcia, D., Illangasekare, T. H., \& Rajaram, H. (2005). Differences in the scale-dependence of dispersivity estimated from temporal and spatial moments in chemically and physically heterogeneous porous media. Advances in Water Resources, 28(7), 745-759. https://doi.org/10.1016/j.advwatres.2004.12.011

Fogg, G. E., \& Zhang, Y. (2016). Debates-Stochastic subsurface hydrology from theory to practice: A geologic perspective. Water Resources Research, 52(12), 9235-9245. https://doi.org/10.1002/2016WR019699

Ganti, V., Singh, A.., Passalacqua, P., \& Foufoula-Georgiu, E. (2009). Subordinated Brownian motion model for sediment transport, Phys. Rev. E, 80, 011111, doi:1539-5663755/2009/80(1)/011111(9)

Gelhar, L. W. (1993). Stochastic Subsurface Hydrology. Prentice-Hall.

Gist, G. A., Thompson, A. H., Katz, A. J., \& Higgins, R. L. (1990). Hydrodynamic dispersion and pore geometry in consolidated rock. Physics of Fluids A: Fluid Dynamics, 2(9), 1533-1544. https://doi.org/10.1063/1.857602

Gómez-Hernández, J. J., \& Wen, X.-H. (1998). To be or not to be multi-Gaussian? A reflection on stochastic hydrogeology. Advances in Water Resources, 21(1), 47-61. https://doi.org/10.1016/S0309-1708(96)00031-0

Guadagnini, A., Riva, M., \& Neuman, S.P. (2012). Extended power-law scaling of heavy-tailed random air-permeability fields in fractured and sedimentary rocks, Hydrol. Earth Syst. Sci., 16, 3249-3260, doi:10.5194/hess-16-3249-2012 
Guadagnini, A., Riva, M., \& Neuman, S. P. (2018). Recent advances in scalable nonGaussian geostatistics: The generalized sub-Gaussian model. Journal of Hydrology, 562, 685-691. https://doi.org/10.1016/j.jhydrol.2018.05.001

Guadagnini, A., Neuman, S.P., Schaap, M.G., \& Riva, M. (2013). Anisotropic statistical scaling of vadose zone hydraulic property estimates near Maricopa, Arizona, Water Resour. Res., 49, doi:10.1002/2013WR014286

Guadagnini, A., S.P. Neuman, M.G. Schaap, and M. Riva (2014), Anisotropic Statistical Scaling of Soil and Sediment Texture in a Stratified Deep Vadose Zone near Maricopa, Arizona, Geoderma, 214-215, 217-227, doi:10.1016/j.geoderma.2013.09.008.

Harbaugh, A.W., 2005. MODFLOW-2005, The U.S. Geological Survey Modular Ground-Water Model - the Ground-Water Flow Process. U.S. Geological Survey Techniques and Methods 6-A16

James, G., Witten, D., Hastie, T., \& Tibshirani, R. (2013). An Introduction to Statistical Learning: With Applications in R. Springer Publishing Company, Incorporated.

Jankovic, I., Maghrebi, M., Fiori, A., \& Dagan, G. (2017). When good statistical models of aquifer heterogeneity go right: The impact of aquifer permeability structures on 3D flow and transport. Advances in Water Resources, 100, 199-211. https://doi.org/10.1016/J.ADVWATRES.2016.10.024

Kitanidis, P. K. (1997). Introduction to Geostatistics: Applications in Hydrogeology. Cambridge University Press. https://doi.org/10.1017/CBO9780511626166

LaBolle, E.M., Quastel, J.,Fogg, G.E., \& Gravner, J. (2000). Diffusion processes in composite porous media and their numerical integration by random walks: Generalized stochastic differential equations with discontinuous coefficients, Water Resour. Res., 36, 3, doi: 10.1029/1999WR900224

Le Borgne, T., Dentz, M., \& E. Villemaux (2013). Stretching, coalescence, and mixing in porous media. Physical Review Letters, 110, 20, doi:10.1103/PhysRevLett.110.204501

Le Borgne, T., Dentz, M., \& E. Villemaux (2015). The lamellar description of mixing in porous media. Journal of Fluid Mechanics, 770, 458-498, doi:10.1017/jfm.2015.117

Libera, A., de Barros, F. P. J., Riva, M., \& Guadagnini, A. (2017). Solute concentration at a well in non-Gaussian aquifers under constant and time-varying pumping schedule. Journal of Contaminant Hydrology, 205, 37-46. https://doi.org/https://doi.org/10.1016/j.jconhyd.2017.08.006

Liu, H. H., \& Molz, F. J. (1997). Comment on "Evidence for non-Gaussian scaling behavior in heterogeneous sedimentary formations" by Scott Painter. Water Resources Research, 33(4), 907-908. https://doi.org/10.1029/96WR03788 
Liu, X., \& Müller, H.-G. (2004). Functional Convex Averaging and Synchronization for Time-Warped Random Curves. Journal of the American Statistical Association, 99(467), 687-699. https://doi.org/10.1198/016214504000000999

Lu, Z., \& Stauffer, P. H. (2012). On estimating functional average breakthrough curve using time-warping technique and perturbation approach. Water Resources Research, 48(5). https://doi.org/10.1029/2011WR011506

Meerschaert, M. M., T. J. Kozubowski, F. J. Molz, and S. Lu (2004), Fractional Laplace model for hydraulic conductivity, Geophys. Res. Lett., 31, L08501, doi:10.1029/2003GL019320

Naff, R. L., Haley, D. F., \& Sudicky, E. A. (1998a). High-resolution Monte Carlo simulation of flow and conservative transport in heterogeneous porous media: 1 . Methodology and flow results. Water Resources Research, 34(4), 663-677. https://doi.org/10.1029/97WR02712

Naff, R. L., Haley, D. F., \& Sudicky, E. A. (1998b). High-resolution Monte Carlo simulation of flow and conservative transport in heterogeneous porous media: 2 . Transport results. Water Resources Research, 34(4), 679-697. https://doi.org/10.1029/97WR02711

Painter, S. (1996). Evidence for Non-Gaussian Scaling Behavior in Heterogeneous Sedimentary Formations. Water Resources Research, 32(5), 1183-1195. https://doi.org/10.1029/96WR00286

Painter, S. (2001), Flexible scaling model for use in random field simulation of hydraulic conductivity, Water Resources Research, 37, 1155-1163. https://doi.org/10.1029/2000WR900394

Panzeri, M., Riva, M., Guadagnini, A., \& Neuman, S. P. (2016). Theory and generation of conditional, scalable sub-Gaussian random fields. Water Resources Research, 52(3), 1746-1761. https://doi.org/10.1002/2015WR018348

Pedretti, D., Fernàndez-Garcia, D., Bolster, D., \& Sanchez-Vila, X. (2013). On the formation of breakthrough curves tailing during convergent flow tracer tests in three-dimensional heterogeneous aquifers. Water Resources Research, 49(7), 4157-4173. https://doi.org/10.1002/wrcr.20330

Pedretti, D., \& Fernàndez-Garcia, D. (2013). An automatic locally-adaptive method to estimate heavily-tailed breakthrough curves from particle distributions. Advances in Water Resources, 59, 52-65. https://doi.org/10.1016/j.advwatres.2013.05.006

Pedretti, D., Masetti, M., \& Beretta, G. Pietro. (2017). Stochastic analysis of the efficiency of coupled hydraulic-physical barriers to contain solute plumes in highly heterogeneous aquifers. Journal of Hydrology, 553, 805-815. https://doi.org/10.1016/j.jhydrol.2017.08.051

Riva, M., Guadagnini, A., Fernàndez-Garcia, D., Sanchez-Vila, X., \& Ptak, T. (2008). Relative importance of geostatistical and transport models in describing heavily 
tailed breakthrough curves at the Lauswiesen site. Journal of Contaminant Hydrology, 101(1-4), 1-13. https://doi.org/10.1016/j.jconhyd.2008.07.004

Riva, M., Guadagnini, A., \& Neuman, S. P. (2017). Theoretical analysis of nonGaussian heterogeneity effects on subsurface flow and transport. Water Resources Research, 53(4), 2998-3012. https://doi.org/10.1002/2016WR019353

Riva, M., Neuman, S. P., \& Guadagnini, A. (2015a). New scaling model for variables and increments with heavy-tailed distributions. Water Resources Research, 51(6), 4623-4634. https://doi.org/10.1002/2015WR016998

Riva, M., Panzeri, M., Guadagnini, A., \& Neuman, S. P. (2015b). Simulation and analysis of scalable non-Gaussian statistically anisotropic random functions. Journal of Hydrology, 531, 88-95. https://doi.org/10.1016/J.JHYDROL.2015.06.066

Riva, M., Neuman, S. P., \& Guadagnini, A. (2013a). Sub-Gaussian model of processes with heavy-tailed distributions applied to air permeabilities of fractured tuff. Stochastic Environmental Research and Risk Assessment, 27(1), 195-207. https://doi.org/10.1007/s00477-012-0576-y

Riva, M., Neuman, S. P., Guadagnini, A., \& Siena, M. (2013b). Anisotropic Scaling of Berea Sandstone Log Air Permeability Statistics. Vadose Zone Journal, 12. https://doi.org/10.2136/vzj2012.0153

Rizzo, C. B., \& de Barros, F. P. J. (2017). Minimum Hydraulic Resistance and Least Resistance Path in Heterogeneous Porous Media. Water Resources Research, 53(10), 8596-8613. https://doi.org/10.1002/2017WR020418

Rubin, Y. (1990a). Stochastic modeling of macrodispersion in heterogeneous porous media. Water Resources Research, 26(1), 133-141. https://doi.org/10.1029/WR026i001p00133

Rubin, Y. (1990b). Correction to "Stochastic modeling of macrodispersion in heterogeneous porous media" by Yoram Rubin. Water Resources Research, 26(10), 2631. https://doi.org/10.1029/WR026i010p02631

Rubin, Y. (2003). Applied stochastic hydrogeology. New York : Oxford University Press.

Salamon, P., Fernàndez-Garcia, D., \& Gómez-Hernández, J. J. (2006). A review and numerical assessment of the random walk particle tracking method. Journal of Contaminant Hydrology, 87(3-4), 277-305. https://doi.org/10.1016/j.jconhyd.2006.05.005

Siena, M., Guadagnini, A., Riva, M., \& Neuman, S. P. (2012). Extended power-law scaling of air permeabilities measured on a block of tuff. Hydrology and Earth System Sciences, 16(1), 29-42. https://doi.org/10.5194/hess-16-29-2012 
Siena, M., \& Riva, M. (2018). Groundwater withdrawal in randomly heterogeneous coastal aquifers. Hydrology and Earth System Sciences, 22(5), 2971-2985. https://doi.org/10.5194/hess-22-2971-2018

Siena, M., Riva, M., Giamberini, M., Gouze, P., \& Guadagnini, A. (2019). Statistical modeling of gas-permeability spatial variability along a limestone core, Spatial Statistics, 39, 100249, doi: 10.1016/j.spasta.2017.07.007.

Siena, M., Guadagnini, A., Bouissonnié, A., Ackerer, P., Daval, D., \& Riva, M. (2020). Generalized Sub-Gaussian processes: theory and application to hydrogeological and geochemical data, Water Resources Research, WRCR24714, doi:10.1029/2020WR027436

Silverman, B. W. (1986). Density Estimation for Statistics and Data Analysis. ChapMan \& HALL/CRC (Vol. 37). https://doi.org/10.2307/2347507

Sole-Mari, G., \& Fernàndez-Garcia, D. (2018). Lagrangian Modeling of Reactive Transport in Heterogeneous Porous Media With an Automatic Locally Adaptive Particle Support Volume. Water Resources Research, 54(10). https://doi.org/10.1029/2018WR023033

Sole-Mari, G., Fernàndez-Garcia, D., Rodríguez-Escales, P., \& Sanchez-Vila, X. (2017). A KDE-Based Random Walk Method for Modeling Reactive Transport With Complex Kinetics in Porous Media. Water Resources Research, 53(11). https://doi.org/10.1002/2017WR021064

Sole-Mari, G., Bolster, D., Fernàndez-Garcia, D., \& Sanchez-Vila, X. (2019). Particle Density Estimation with Grid-Projected and Boundary-Corrected Adaptive Kernels. Advances in Water Resources, 103382. https://doi.org/10.1016/j.advwatres.2019.103382

Winter, C. L., Tartakovsky, D. M., \& Guadagnini, A. (2003). Moment Differential Equations for Flow in Highly Heterogeneous Porous Media. Surveys in Geophysics, 24(1), 81-106. https://doi.org/10.1023/A:1022277418570

Winter, C. L., \& Tartakovsky, D. M. (2000). Mean Flow in composite porous media. Geophysical Research Letters, 27(12), 1759-1762. https://doi.org/10.1029/1999GL011030

Winter, C. L., \& Tartakovsky, D. M. (2002). Groundwater flow in heterogeneous composite aquifers. Water Resources Research, 38(8), 11-23. https://doi.org/10.1029/2001WR000450

\section{Appendix A: Analytical formulation of the GSG model and key properties}

Riva et al. (2015a,b) introduce the Generalized Sub-Gaussian (GSG) model according to which the random function $Y^{\prime}(\mathbf{x})$ defined by (1) is described considering the 
934 subordinator $U(\mathbf{x})$ to be lognormally distributed according to $\ln (U) \sim \mathcal{N}\left[0,(2-\alpha)^{2}\right]$, 935 i.e.,

936

$$
f_{U}(u)=\frac{e^{-\frac{(\ln u)^{2}}{2(2-\alpha)^{2}}}}{\sqrt{2 \pi} u(2-\alpha)}
$$

937 with $\alpha<2$. Here, we include a brief summary of the key analytical formulations and 938 scaling properties of the isotropic GSG model introduced by Riva et al (2015a). 939 Corresponding formulations for anisotropic fields have been derived by Riva et al 940 (2015b).

941 The marginal $p d f$ of $Y^{\prime}(\mathbf{x}), f_{Y^{\prime}}\left(y^{\prime}\right)$, is fully defined by $\alpha$ and the standard deviation 942

$$
f_{Y^{\prime}}\left(y^{\prime}\right)=\frac{1}{2 \pi \sigma_{G}(2-\alpha)} \int_{0}^{\infty} e^{-\frac{1}{2}\left[\left(\frac{\ln u}{2-\alpha}\right)^{2}+\left(\frac{y}{\sigma_{G} u}\right)^{2}\right]} \frac{d u}{u^{2}} .
$$

The latter corresponds to a normal-lognormal (NLN) distribution whose variance, $\sigma_{Y}^{2}$, and standardized kurtosis, $\kappa_{Y^{\prime}}$, of $Y^{\prime}(\mathbf{x})$ are given by

$$
\sigma_{Y}^{2}=\sigma_{G}^{2} e^{2(2-\alpha)^{2}}, \quad \kappa_{Y^{\prime}}=3 e^{4(2-\alpha)^{2}}
$$

It is noted that the lognormal distribution (A1) tends to a delta function when $\alpha \rightarrow$ 2 and (A2) reduces to the Gaussian distribution. Figure A1 depicts $f_{Y}$, for the three values of $\alpha$ examined in our study and by setting $\sigma_{Y}^{2}=1$. The Gaussian $p d f$ having the same mean and variance as $Y^{\prime}(\mathbf{x})$ and corresponding to $\alpha \rightarrow 2$ is also depicted for completeness. While $f_{Y^{\prime}}$ is close to the Gaussian $p d f$ for $\alpha=1.8$, one can observe that $f_{Y^{\prime}}$ exhibits peaks and tails which become sharp and long, respectively, as $\alpha$ decreases. These features, which represent a clear deviation from a Gaussian distribution, is also quantified by the excess kurtosis, $E K_{Y^{\prime}}=\kappa_{Y^{\prime}}-3$, whose value increases (deviating from zero, which corresponds to the scenario for which $\alpha \rightarrow 2$ ) as $\alpha$ decreases. 


$$
f_{\Delta Y}(\Delta y)=\frac{1}{2 \pi \sigma_{G}(2-\alpha)^{2} \sqrt{2 \pi}} \int_{0}^{\infty} \int_{0}^{\infty} e^{-\frac{1}{2}\left[\frac{\ln ^{2} u_{1}+\ln ^{2} u_{2}}{(2-\alpha)^{2}}+\frac{\Delta y^{2}}{\sigma_{G}^{2} r^{2}}\right]} \frac{d u_{2} d u_{1}}{u_{2} u_{1} r}
$$

959

960

961

962

963

964

965

966

967

968

969

970

971

972

973

974

975

976

977

978

979

980

981

982

Here, $r=\sqrt{u_{1}^{2}+u_{2}^{2}-2 \rho_{G} u_{1} u_{2}}$ and $\rho_{G}(s)$ is the correlation function of $G(\mathbf{x})$ evaluated at separation distance (or lag) $s=|s|$. The standardized kurtosis of $\Delta Y$ is rendered as

$$
\kappa_{\Delta Y}=3 \mathrm{e}^{2(2-\alpha)^{2}}\left[1+\frac{1}{2}\left(\frac{e^{2(2-\alpha)^{2}}-1}{e^{(2-\alpha)^{2}}-\rho_{G}}\right)^{2}\right]
$$

It is remarked that the dependence of $\rho_{G}(s)$ on lag induces a corresponding dependence of the shape of $f_{\Delta Y}$. For example, $\kappa_{\Delta Y} \rightarrow 3$ and the distribution of $\Delta Y$ tends to the Gaussian one as $\alpha \rightarrow 2$. Otherwise, the shape of $f_{\Delta Y}$ scales with lag (or, equivalently, with $\rho_{G}$ ) with peak which tends to sharpen and tails to become heavier (i.e., corresponding to increased $\kappa_{\Delta Y}$ ) as a function of $\rho_{G}$. Figure A2 depicts $f_{\Delta Y}$ for three exemplary lags and $\alpha=1.8$ (Fig. A2a), 1.5 (Fig. A2b), and 1.2 (Fig. A2c). All GSG fields are characterized by the same variance $\left(\sigma_{Y}^{2}=1\right)$ and integral scale $\left(I_{Y}=I_{G} e^{-(2-\alpha)^{2}}, I_{G}\right.$ being the integral scale of $G(\mathbf{x})$ ), and lags are normalized with respect to $I_{Y}$. Also shown for comparison are Gaussian distributions having the same variance as $\Delta Y$. In all cases, $f_{\Delta Y}$ exhibits sharp peaks and heavy tails at small lags. Increasing lag, $f_{\Delta Y}$ tends to become Gaussian when $\alpha$ is large (i.e., $\alpha=1.8$ ), otherwise remaining heavy tailed for smaller values of $\alpha$. This feature is also illustrated in Figure A3, depicting excess kurtosis of $Y^{\prime}$ and $\Delta Y$ versus lag for the three values of $\alpha$ considered in our study. The excess kurtosis of $\Delta Y, E K_{\Delta Y}=\kappa_{\Delta Y}-3$, decreases as lag increases, rendering the peak of $f_{\Delta Y}$ less sharp and its tails lighter. When $\alpha=1.8, E K_{\Delta Y}$ is seen to tend to an asymptotic value which is $<<1$, so that $f_{\Delta Y}$ becomes virtually Gaussian. Included in Figure A3 are horizontal lines depicting the excess of kurtosis of $Y^{\prime}, E K_{Y^{\prime}}$. In all investigated cases, $E K_{Y^{\prime}}>E K_{Y^{\prime}}$ at small lags, i.e. $f_{\Delta Y}$ has sharper peaks and heavier tails than does $f_{Y^{\prime}}$, the opposite being true at large lags.

The variogram, $\gamma_{Y}$, of $Y^{\prime}$, is given by

$$
\gamma_{Y}=\sigma_{G}^{2} e^{(2-\alpha)^{2}}\left(e^{(2-\alpha)^{2}}-1\right)+e^{(2-\alpha)^{2}} \gamma_{G}
$$


$983 \gamma_{G}$ being the variogram of $G(\mathbf{x})$. Note that according to (A6) $\gamma_{Y}$ is discontinuous at the 984 origin, i.e., at $s=0$, thus exhibiting a nugget effect. Figure A4 compares (on arithmetric 985 and logarithm scales) $\gamma_{Y}$ computed with three values of $\alpha(=1.2,1.5$, and 1.8) and setting $986 \sigma_{Y}^{2}=1$ and $I_{Y}=1$. Also shown for comparison is the variogram obtained within a 987 Gaussian field (i.e., corresponding to $\alpha \rightarrow 2$ ) characterized by the same variance and 988 integral scale as $Y^{\prime}$ and by an exponential correlation function. The variogram associated 989 with $\alpha=1.8$ virtually coincides with its counterpart associated with a Gaussian field 990 everywhere, with the exception of small lags where the GSG variogram is characterized 991 by a nugget (clearly visible on logarithm scale).

992

993

994

995

996

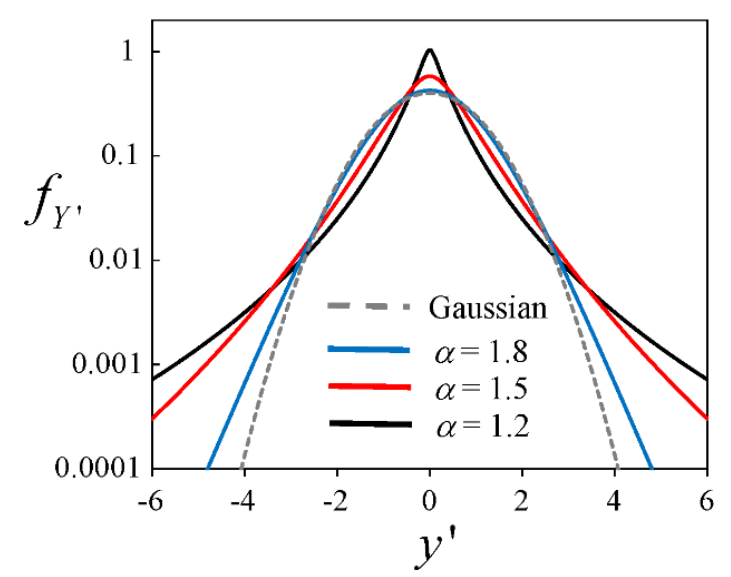

Figure A1: Probability density function of $Y^{\prime}$ for $\sigma_{Y}^{2}=1$ and three values of $\alpha$ (solid curves). Also shown is the Gaussian pdf having the same mean and variance as $Y^{\prime}$ (dashed curve). 

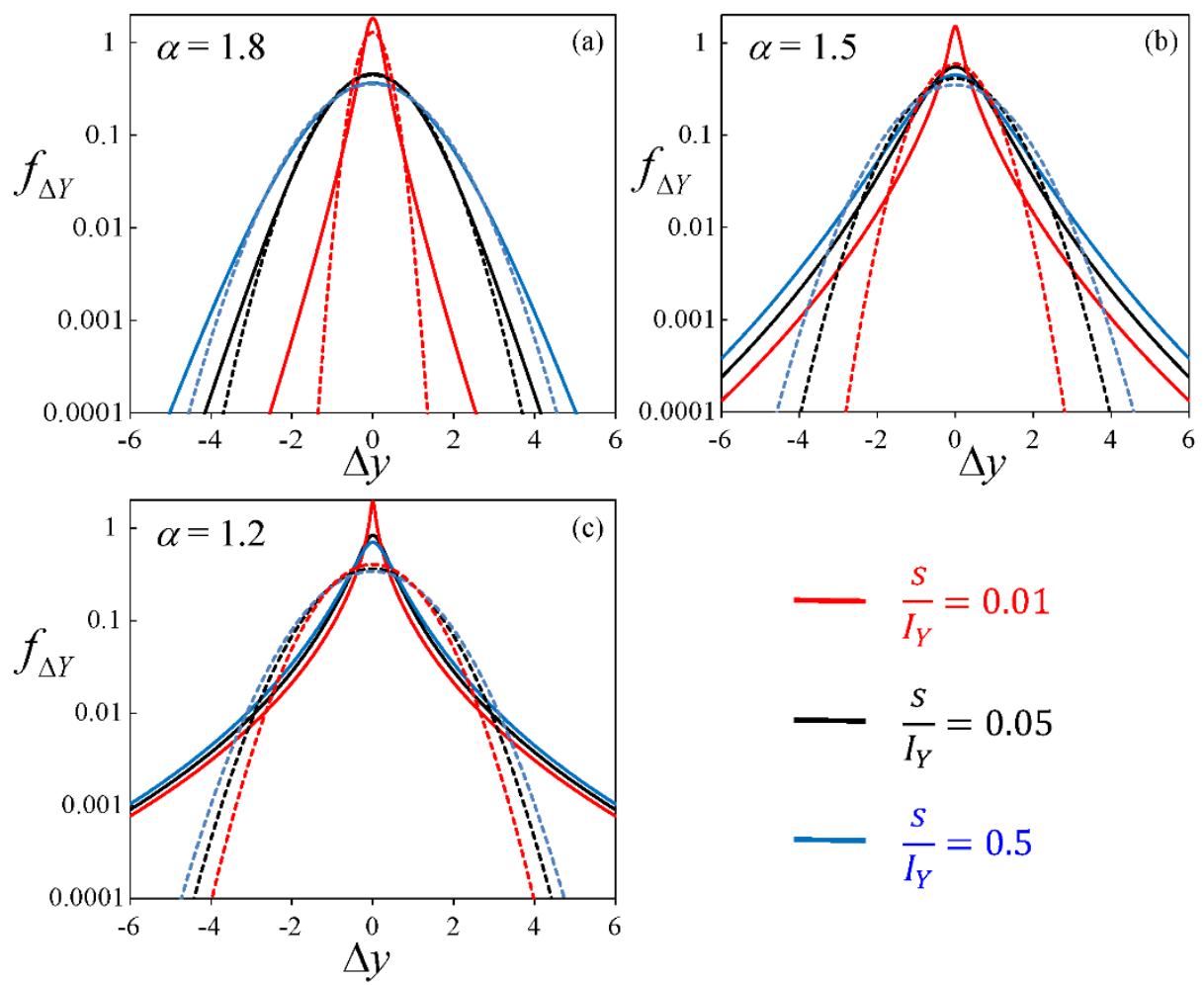

$$
\begin{aligned}
-\frac{S}{I_{Y}} & =0.01 \\
-\frac{S}{I_{Y}} & =0.05 \\
-\frac{S}{I_{Y}} & =0.5
\end{aligned}
$$

Figure A2: Probability density function of $\Delta Y$ for $\sigma_{Y}^{2}=1$, three lags and (a) $\alpha=1.8$, (b) as $\Delta Y$ (dashed curves). Lags are normalized with respect to $I_{Y}$.

1002

1003

1004

1005

1006

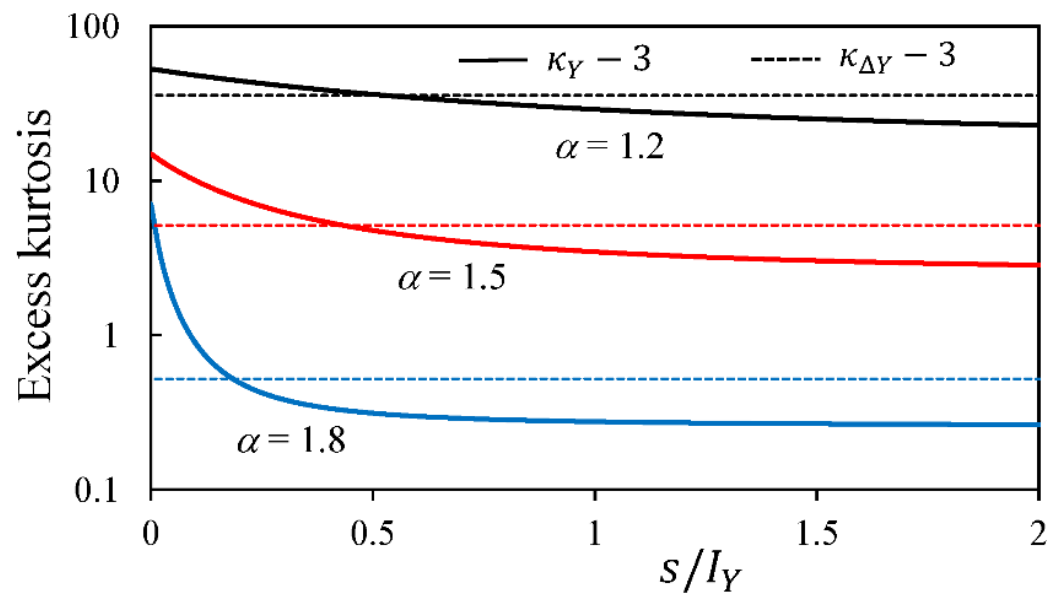

Figure A3: Excess kurtosis of $Y^{\prime}$ (dashed horizontal lines) and $\Delta Y$ (solid curves) versus normalized lag for $\alpha=1.2,1.5$, and 1.8 . 
1007

1008

1009

1010

1011

1012

1013

1014

1015

1016

1017

1018

1019

1020

1021

1022

1023

1024

1025
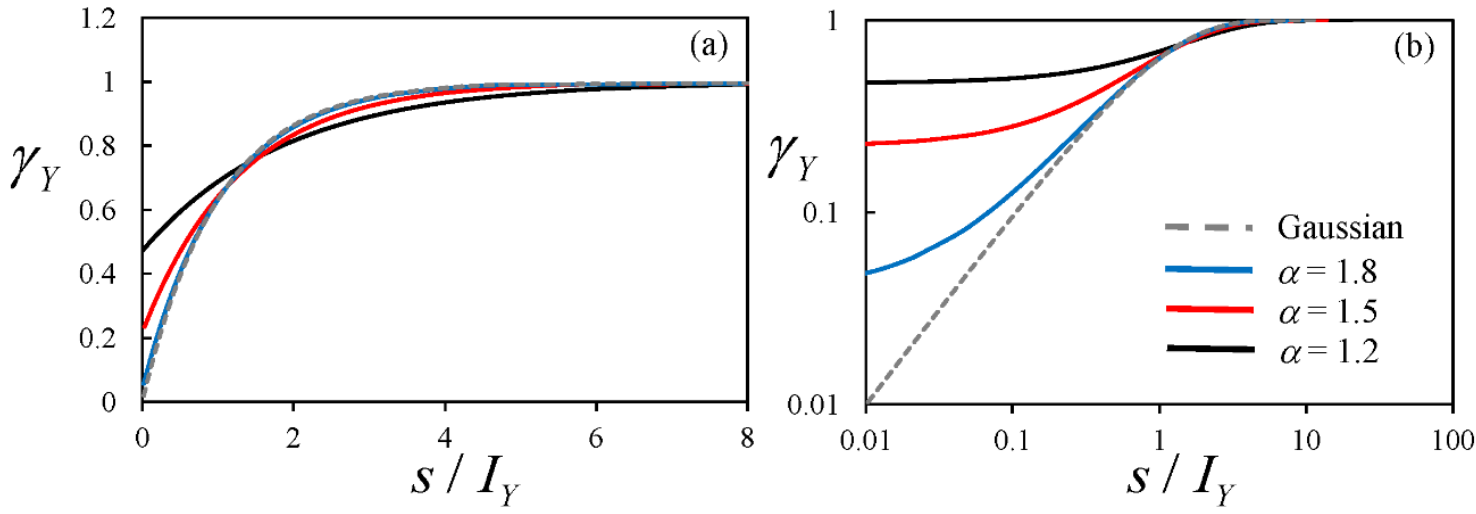

Figure A4: Variogram of $Y^{\prime}$ obtained for ${ }_{\alpha}=1.2,1.5$, and 1.8 on (a) arithmetic and (b) logarithmic scales (solid curves). Also shown for comparison the variogram associated with a Gaussian field (and corresponding to $\alpha \rightarrow 2$ ) characterized by the same variance and integral scale as $Y^{\prime}$ (dashed curve).

\section{Appendix B: Transport Metrics}

\section{B.1. Percentile average of concentration curves}

We obtain percentile-averaged curves over all MC realizations of $(i)$ sectionintegral longitudinal profiles of concentration at a given observation time and (ii) temporal distributions of mass flux through a given horizontal plane (or BTCs), here denoted as $\bar{C}$ and $\bar{J}$, respectively. Following the terminology used by Lu and Stauffer (2012) for BTCs, the percentile average results from averaging over the times of the cumulative distribution of $\bar{J}$ at fixed percentiles. By analogy, here we also extend this technique to longitudinal concentration profiles.

The first step of the procedure is to rearrange in ascending order the collection of particle positions / arrival times corresponding to each realization, i.e.,

$$
\begin{aligned}
& Z_{p, r}\left(t_{i}\right)<Z_{p+1, r}\left(t_{i}\right), \quad \forall p \in\{1,2, \ldots,(n-1)\}, \\
& T_{p, r}\left(z_{j}\right)<T_{p+1, r}\left(z_{j}\right), \quad \forall p \in\{1,2, \ldots,(n-1)\},
\end{aligned}
$$


1027

1028

1029

1030

1031

1032

1033

1034

1035

1036

1037

1038

1039

1040

1041

1042

1043

in (11), each particle index $p$ is associated with a value of $\left\langle Z_{p}\left(t_{i}\right)\right\rangle$ or $\left\langle T_{p}\left(z_{j}\right)\right\rangle$. According to the above-mentioned definition of percentile average, these values correspond to regular increments in the cumulative (i.e., integral) forms of $\bar{C}\left(z, t_{i}\right)$ and $\bar{J}\left(z_{j}, t\right)$, i.e.,

$$
\begin{gathered}
\int_{0}^{z} \bar{C}\left(\zeta, t_{i}\right) \mathrm{d} \zeta=\frac{1}{n} \sum_{p=1}^{n} \mathcal{H}\left(\left\langle Z_{p}\left(t_{i}\right)\right\rangle-z\right), \\
\int_{0}^{t} \bar{J}\left(z_{j}, \tau\right) \mathrm{d} \tau=\frac{1}{n} \sum_{p=1}^{n} \mathcal{H}\left(\left\langle T_{p}\left(z_{j}\right)\right\rangle-t\right),
\end{gathered}
$$

where $\mathcal{H}$ is the Heaviside function. Differentiation of (B3) and (B4) yields

$$
\begin{aligned}
& \bar{C}\left(z, t_{i}\right)=\frac{1}{n} \sum_{p=1}^{n} \delta\left(\left\langle Z_{p}\left(t_{i}\right)\right\rangle-z\right), \\
& \bar{J}\left(z_{j}, t\right)=\frac{1}{n} \sum_{p=1}^{n} \delta\left(\left\langle T_{p}\left(z_{j}\right)\right\rangle-t\right),
\end{aligned}
$$

where $\delta$ is the Dirac delta. Since $n$ is a finite number, these expressions need to be smoothed to produce adequate estimates. This is accomplished by replacing $\delta$ in (B5) (B6) with a smoothing kernel $W$ characterized by a smoothing bandwidth $h$. This constitutes the basis of Kernel Density Estimation (KDE), which is summarized in Appendix D.

\section{B.2. Moments}

We study the evolution of the spatial and temporal moments of the section-integral concentrations $\left(C_{r}\right)$ and the breakthrough curves $\left(J_{r}\right)$, respectively, in each realization $r$, as rendered through 
$1044 \quad \mu_{1, r}^{\mathrm{S}}\left(t_{i}\right)=\int_{0}^{L_{z}} z C_{r}\left(z, t_{i}\right) \mathrm{d} z, \quad \mu_{\kappa, r}^{\mathrm{S}}(t)=\int_{0}^{L_{z}}\left(z-\mu_{1}^{\mathrm{S}}\left(t_{i}\right)\right)^{\kappa} C_{r}\left(z, t_{i}\right) \mathrm{d} z, \quad \kappa \geq 2,(\mathrm{~B} 7)$

$1045 \quad \mu_{1, r}^{\mathrm{T}}\left(z_{j}\right)=\int_{0}^{\infty} t J_{r}\left(z_{j}, t\right) \mathrm{d} t, \quad \mu_{\kappa, r}^{\mathrm{T}}(z)=\int_{0}^{\infty}\left(t-\mu_{1}^{\mathrm{T}}\left(z_{j}\right)\right)^{\kappa} J_{r}\left(z_{j}, t\right) \mathrm{d} t, \quad \kappa \geq 2$, (B8) 1046

where $\kappa$ is the moment order, and superscripts S and T stand for spatial and temporal, 1047 respectively. In practice, integrals in (B7) and (B8) are estimated directly from the 1048 discrete particle positions and arrival times, respectively, as

$$
\mu_{1, r}^{\mathrm{S}}\left(t_{i}\right)=\frac{1}{n} \sum_{p=1}^{n} Z_{p, r}\left(t_{i}\right), \quad \mu_{\kappa, r}^{\mathrm{S}}\left(t_{i}\right)=\frac{1}{n} \sum_{p=1}^{n}\left[Z_{p, r}\left(t_{i}\right)-\mu_{1, r}^{\mathrm{S}}\left(t_{i}\right)\right]^{\kappa}, \quad \kappa \geq 2,
$$

$$
\mu_{1, r}^{\mathrm{T}}\left(z_{j}\right)=\frac{1}{n} \sum_{p=1}^{n} T_{p, r}\left(z_{j}\right), \quad \mu_{\kappa, r}^{\mathrm{T}}\left(z_{j}\right)=\frac{1}{n} \sum_{p=1}^{n}\left[T_{p, r}\left(z_{j}\right)-\mu_{1, r}^{\mathrm{T}}\left(z_{j}\right)\right]^{\kappa}, \quad \kappa \geq 2 .(\mathrm{B} 10)
$$
$\kappa=4)$

$$
\tilde{\mu}_{\kappa, r}^{\mathrm{S}}\left(t_{i}\right)=\frac{\mu_{\kappa, r}^{\mathrm{S}}\left(t_{i}\right)}{\left[\mu_{2, r}^{\mathrm{S}}\left(t_{i}\right)\right]^{\frac{\kappa}{2}}}, \quad \tilde{\mu}_{k, r}^{\mathrm{T}}\left(z_{j}\right)=\frac{\mu_{\kappa, r}^{\mathrm{T}}\left(z_{j}\right)}{\left[\mu_{2, r}^{\mathrm{T}}\left(z_{j}\right)\right]^{\frac{\kappa}{2}}}, \quad \kappa \geq 3 .
$$

We noted that temporal moments computed in our study from the full set of particle arrival times (see (B10)) were mainly controlled by the tails of the associated distributions. BTCs in heterogeneous porous media often display heavy tails that decay 1057 slowly with a power-law behavior (e.g., Pedretti et al., 2013). Even truncated power laws may have very high values of higher-order moments that would hardly be accurately inferred from simulations with a reasonable number of particles. To overcome this issue, temporal moments were computed by excluding the $1 \%$ highest arrival times. 

times $t_{i}$ and all evaluation positions $z_{j}$, respectively:

1064

$$
\frac{\partial \mu_{1, r}^{\mathrm{S}}}{\partial t}\left(t_{i}\right) \geq \bar{v}, \quad \mu_{1, r}^{\mathrm{T}}\left(z_{j}\right)=\frac{z_{j}}{\bar{v}}
$$

1065

1066

1067

1068

1069

1070

1071

1072

\section{B.3. Key arrival times}

As a complement to temporal moments, we evaluate some additional BTC features that may provide further insight on transport behavior. Namely, we compute: the first arrival time $\left(T_{1, r}\right)$, the arrival time corresponding to the peak of the corresponding concentration curve $\left(T_{\mathrm{peak}, r}\right)$ and the $99^{\text {th }}$ percentile arrival time $\left(T_{\mathrm{p} 99, r}\right)$. The peak is identified in each realization $r$ from the KDE reconstruction of the BTC (see Appendix D), whereas the two other measures are obtained directly from particle arrival times.

\section{B.4. Advective stretching}

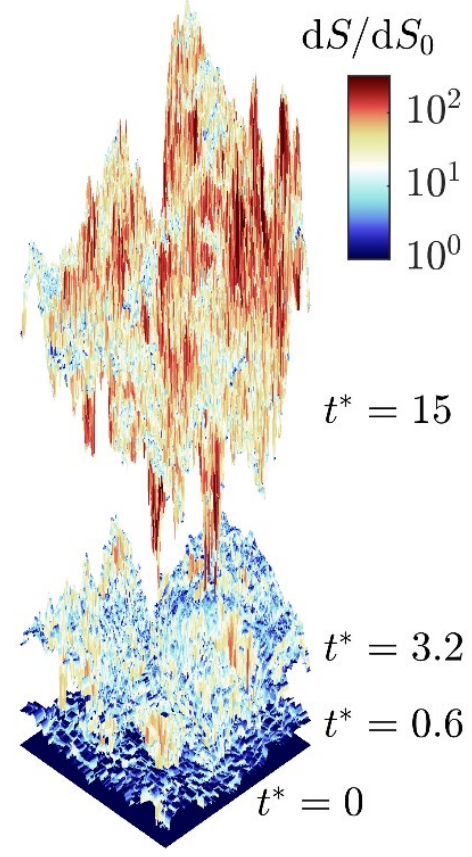
heterogeneous advection, and mapping of the local surface growth. 

of triangular surfaces defined by the initial relative position of particles in the scenario

1079

1080

1081

1082

1083

1084

1085

1086

1087

1088

1089

1090

1091

1092

1093

1094

1095

1096

1097

1098

1099

corresponding to $\mathrm{Pe} \rightarrow \infty$. Figure B1 illustrates the local growth of the individual

triangular surfaces $\mathrm{d} S$ at three observation times in a selected realization of $Y$. In Section

3 we focus on the ratio $S / S_{0}$, where $S$ is the sum of all triangular $\mathrm{d} S$, and $S_{0}=L_{x} L_{y}$.

\section{Appendix C: Quantification of Distribution Overlaps}

Here, we provide a synthesis of the basic concepts of Bayesian classification, focusing on the methods we employ in our study.

\section{C.1. The Bayes classifier}

The Bayes classifier assigns observations of a (multi-dimensional random) vector $\mathbf{R}$, with values $\mathbf{r}$, to a class $K$ (which has a finite number $N_{\mathrm{k}}$ of possible values $k$ ) according to data-driven estimates of the conditional probability density functions $f_{k}(\mathbf{r})=p(\mathbf{R}=\mathbf{r} \mid K=k)$. Ideally, it is the classification method with the least associated error provided that the true $p d f \mathrm{~s}, f_{k}$, are known or reproduced correctly. Given an observation with a value $\mathbf{r}$, this method assigns it to the category $k$ for which $g_{k}(\mathbf{r})=$ $P(K=k \mid \mathbf{R}=\mathbf{r})$ is highest among all possible $k$. According to Bayes theorem

$$
g_{k}(\mathbf{r})=\frac{p_{k} f_{k}(\mathbf{r})}{\sum_{\ell=1}^{N_{\mathrm{k}}} p_{\ell} f_{\ell}(\mathbf{r})}
$$

where $p_{k}=P(K=k)$ is the prior probability of any observation to belong to class $k$. If no prior information is available, all $p_{\ell}=p_{k}(\forall \ell \neq k)$, and the classifier will assign the observation $\mathbf{r}$ to the class $k$ for which $f_{k}$ is highest. If we assume that all $f_{k}$ are Gaussian, with mean $\widehat{\mathbf{m}}_{k}$ and covariance matrix $\widehat{\boldsymbol{\Sigma}}_{k}$ estimated from data, then the classification is equivalent to assigning observation $\mathbf{r}$ to the class $k$ for which

$$
\delta_{k}(\mathbf{r})=-\left(\mathbf{r}-\widehat{\mathbf{m}}_{k}\right)^{\mathrm{T}} \widehat{\boldsymbol{\Sigma}}_{k}^{-1}\left(\mathbf{r}-\widehat{\mathbf{m}}_{k}\right)-\log \left|\widehat{\boldsymbol{\Sigma}}_{k}\right|+2 \log p_{k}
$$


1100

1101

1102

1103

1104

1105

is highest. This approach is known as Quadratic Discriminant Analysis (QDA).

For one-dimensional variables, $R$, it may in some cases be feasible to reconstruct $f_{k}(r)$ from data, instead of assuming that it is Gaussian. We do so in our study upon relying on Kernel Density Estimation (see Appendix D). Each observation $r$ is then assigned to the class $k$ with the highest $g_{k}(r)$ (see (C1)). We term this approach as $K D E$ discriminant.

\section{C.2. Confusion matrix and Overlap matrix}

In discriminant analysis, the Confusion Matrix compares the obtained classification (rows) with the actual classes (columns) of a number of random observations, so that the entries on the diagonal of the matrix correspond to correct classifications. Normalization of the columns by the total number of observations of each class yields a normalized confusion matrix. The latter informs us on the likelihood of correct (diagonal) and incorrect (outside diagonal) classification. In other words, if we define variable $\widehat{K}$ as the estimated class (and $K$ as the true class), the entries of the normalized confusion matrix are $P_{\ell k}^{\prime}=P(\widehat{K}=\ell \mid K=k)$.

We define the overlap of a $p d f$, $f_{k}$, (conditioned to $K=k$ ) with $f_{\ell}$ (conditioned to $K=\ell)$ as the likelihood that, if the class given by the discriminant analysis, $\widehat{K}$, is $\ell$, the actual class, $K$, is $k$; i.e., $P_{k \ell}=P(K=k \mid \widehat{K}=\ell)$. The quantity $P_{k \ell}$ (and in particular, its values for $k=\ell$ ) indicates the level of reliability associated with relying on the $p d f s$ to estimate the actual class of an individual realization. Mapping $P_{\ell k}^{\prime}$ onto $P_{k \ell}$ is accomplished through Bayes theorem

$$
P_{k \ell}=\frac{p_{k} P_{\ell k}^{\prime}}{\sum_{m=1}^{N_{\mathrm{k}}} p_{m} P_{\ell m}^{\prime}}
$$




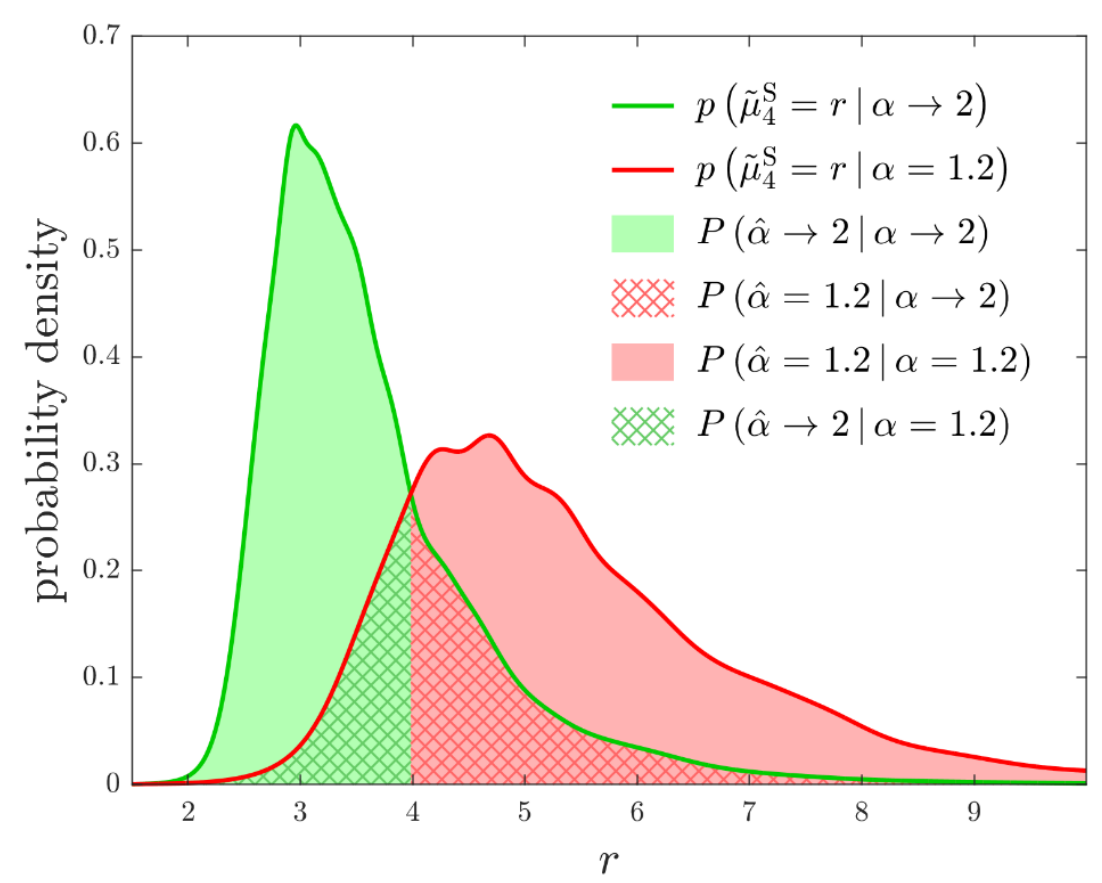

Figure C1: Graphical depiction exemplifying the integration procedure for the evaluation of the $P_{\ell k}^{\prime}$ entries of the normalized confusion matrix using the KDE classifier for the early-time spatial kurtosis.

In our study, the classes are the four studied values of $\alpha$, and the random observations are the selected metrics evaluated from the solute transport results. We use the KDE discriminant for the analysis of each of these metrics, whereas we apply QDA for the joint analysis of several metrics. In both cases, the final result of interest is the overlap matrix defined in Section C.2, obtained from the normalized confusion matrix by (C3). In the case of the KDE discriminant, the entries of the normalized confusion matrix can be obtained by numerical integration of the relevant parts of the $p d f$ s (see Figure $\mathrm{C} 1$ as a graphical example), whereas the confusion matrix for the QDA is obtained by explicitly classifying all observations.

Note that the KDE approach and the QDA approach require a training dataset (for the $\mathrm{KDE}$ reconstruction of the $p d f$ in the former and for the estimation of the mean and 
covariance in the latter). Relying on the same dataset for training and for testing (i.e., for building the confusion matrix) could slightly bias the results towards correct classification (that is, towards a non-overlap result). However, we expect this effect to be negligible given the rather large size of the dataset (i.e., 5000 observations of each class).

\section{Appendix D: Kernel Density Estimation}

Here, we provide a brief introduction to univariate Kernel Density Estimation (KDE), which we use to reconstruct curves, $f(x)$, which are (or can be treated as) probability density functions, relying on a limited number of samples $X_{p}, p=1, \ldots, n$. The KDE is formulated as

$$
f(x) \cong \hat{f}(x)=\frac{1}{n} \sum_{p=1}^{n} W\left(X_{p}-x ; h\right)
$$

where $W$ is a kernel or smoothing function, which we choose to be Gaussian, i.e.,

$$
W(u ; h)=\frac{1}{\sqrt{2 \pi} h} \exp \left(-\frac{u^{2}}{2 h^{2}}\right)
$$

$h$ being the kernel bandwidth.

When $X_{p}$ are particle longitudinal positions or arrival times, $f(x)$ is the longitudinal density of solute mass (8) or the breakthrough curve (9), respectively (Fernàndez-Garcia and Sànchez-Vila, 2011; Sole-Mari et al., 2017, 2019; Sole-Mari and Fernàndez-Garcia, 2018; Pedretti and Fernàndez-Garcia, 2013). Alternatively, the collection of samples $X_{p}$ can be formed by evaluations of a scalar metric that varies across $\mathrm{MC}$ realizations and in this case $f(x)$ is the probability density function of the metric $x$ (see Appendix C).

A key aspect of the accuracy of KDE is to select an optimal value for the bandwidth h. A widely popular technique employed for this aim relies on minimizing the Asymptotic 
1161 Mean Integrated Squared Error (AMISE). It can be shown that, for a Gaussian $W$, in the 1162 limit when $n h \rightarrow \infty$ and $h \rightarrow 0$ (e.g. Silverman, 1986)

1163

$$
\operatorname{AMISE} \approx(2 \sqrt{\pi} n h)^{-1}+\frac{h^{4}}{4} \int_{\mathbb{R}}\left(\frac{\partial^{2} f}{\partial x^{2}}\right)^{2} d x
$$

1164 By setting $\frac{\partial \text { AMISE }}{\partial h}=0$, the absolute minimum AMISE can be shown to be obtained for the 1165 following choice of bandwidth

1166

$$
h=\left[2 \sqrt{\pi} n \int_{\mathbb{R}}\left(\frac{\partial^{2} f}{\partial x^{2}}\right)^{2} \mathrm{~d} x\right]^{-\frac{1}{5}} .
$$

1167 Since $\int_{\mathbb{R}}\left(\frac{\partial^{2} f}{\partial x^{2}}\right)^{2} \mathrm{~d} x$ is a priori unknown, several iterative methodologies have been 1168 developed to estimate it from the data and solve equation (D4). Here, we apply one such 1169 methodology, namely the Improved Sheater-Jones (ISJ) algorithm developed by Botev et 1170 al. (2010). 